\title{
Laser-driven quantum magnonics and terahertz dynamics of the order parameter in antiferromagnets
}

\author{
D. Bossini* \\ Institute for Molecules and Materials, Radboud University, 135 Heyendaalseweg, 6525 AJ Nijmegen, The Netherlands \\ and Experimentelle Physik VI, Technische Universität Dortmund, D-44221 Dortmund, Germany \\ S. Dal Conte and G. Cerullo \\ Dipartimento di Fisica, Politecnico di Milano, Piazza Leonardo da Vinci 32, Milano, Italy \\ and Istituto di Fotonica e Nanotecnologie, Consiglio Nazionale delle Ricerche, Piazza Leonardo da Vinci 32, Milano, Italy \\ O. Gomonay \\ Institut für Physik, Johannes Gutenberg Universität Mainz, D-55099 Mainz, Germany \\ and National Technical University of Ukraine "KPI”, 03056, Kyiv, Ukraine \\ R. V. Pisarev \\ Ioffe Physical-Technical Institute, Russian Academy of Sciences, 194021 St. Petersburg, Russia \\ M. Borovsak and D. Mihailovic \\ Jozef Stefan Institute \& CENN-Nanocenter, Jamova 39, Ljubljana SI-1000, Slovenia \\ J. Sinova \\ Institut für Physik, Johannes Gutenberg Universität Mainz, D-55099 Mainz, Germany \\ and Institute of Physics ASCR, v.v.i., Cukrovarnicka 10, 16253 Praha 6 Czech Republic \\ J. H. Mentink, Th. Rasing, and A. V. Kimel \\ Institute for Molecules and Materials, Radboud University, 135 Heyendaalseweg, 6525 AJ Nijmegen, The Netherlands
}

(Received 26 September 2017; revised manuscript received 2 April 2019; published 25 July 2019)

\begin{abstract}
The impulsive generation of two-magnon modes in antiferromagnets by femtosecond optical pulses, so-called femto-nanomagnons, leads to coherent longitudinal oscillations of the antiferromagnetic order parameter that cannot be described by a thermodynamic Landau-Lifshitz approach. We argue that this dynamics is triggered as a result of a laser-induced modification of the exchange interaction. In order to describe the oscillations, we have formulated a quantum mechanical description in terms of magnon pair operators and coherent states. Such an approach allowed us to derive an effective macroscopic equation of motion for the temporal evolution of the antiferromagnetic order parameter. An implication of the latter is that the photoinduced spin dynamics represents a macroscopic entanglement of pairs of magnons with femtosecond period and nanometer wavelength. By performing magneto-optical pump-probe experiments with 10 femtosecond resolution in the cubic $\mathrm{KNiF}_{3}$ and the uniaxial $\mathrm{K}_{2} \mathrm{NiF}_{4}$ collinear Heisenberg antiferromagnets, we observed coherent oscillations at the frequency of 22 and $16 \mathrm{THz}$, respectively. The detected frequencies as a function of the temperature fit the two-magnon excitation up to the Néel point. The experimental signals are described as dynamics of magnetic linear dichroism due to longitudinal oscillations of the antiferromagnetic vector.
\end{abstract}

DOI: 10.1103/PhysRevB.100.024428

\section{INTRODUCTION}

The research area of ultrafast laser-induced spin dynamics started two decades ago with the observation of subpicosecond demagnetization of $\mathrm{Ni}$ by 60 -femtosecond laser pulses [1] and the subsequent observation of the laser-induced ferromagnetic [2] and antiferromagnetic resonance [3] in the

*davide.bossini@tu-dortmund.de time domain, triggered by laser-induced heating and optically generated effective magnetic field [4-7]. These experiments opened up new opportunities for the generation and the control of propagating spin waves with subpicosecond temporal resolution [8-10]. It even ignited a surge of interest in the field of photomagnonics promising to develop magnon-based information processing into the terahertz (THz) domain [11].

On the fundamental side, driving spins out of equilibrium with femtosecond laser pulses is expected to launch dynamics beyond the realm of classical mechanics and thermodynamics 
[12]. Nevertheless, all the manifestations of light-induced (sub)picosecond spin dynamics have been hitherto interpreted by means of classical equations of motion [4,5,13-16]. This approach was proven to be successful if the photogenerated single-magnon modes have wave vectors near the center of the Brillouin zone.

It is well known that nonzero wave-vector magnons can be optically excited via two-magnon (2M) processes. Obeying the laws of conservation of energy and momentum a photon with the energy $\hbar \omega_{p 1}$ and momentum $k_{p 1}$ can generate two magnons with energies $\hbar \omega_{m 1}, \hbar \omega_{m 2}$ and momenta $k_{m 1}, k_{m 2}$ via the Raman scattering process. As a result, the photon is scattered with the energy $\hbar \omega_{p 2}$ and momentum $k_{p 2}$ so that $\hbar \omega_{p 1}=\hbar \omega_{m 1}+\hbar \omega_{m 2}+\hbar \omega_{p 2}$ and $k_{p 1}=k_{m 1}+k_{m 2}+k_{p 2}$. For visible light and magnons far away from the center of the Brillouin zone, it can be safely assumed that $k_{m 1,2} \gg k_{p 1,2}$. Consequently, light generates two counter-propagating magnons $k_{m 1} \approx k_{m 2}$ and $\omega_{m 1} \approx \omega_{m 2}$. The dominating light-matter process is the interaction of the electric field of light with electric charges; according to the selection rules of electric dipole transitions the total spin in the excitation of the $2 \mathrm{M}$ mode is conserved (see Fig. 1). An effective generation of magnon pairs at the edges of the Brillouin zone, where the magnon density of states is the largest, was demonstrated via spontaneous Raman (SR) scattering [17-22]. Femtosecond laser pulses and the mechanism of impulsive stimulated Raman scattering (ISRS) [23-25,49] led to the generation of pairs of magnons and to the observation of the subsequent spin dynamics in time-domain with temporal resolution on the order of 10 femtoseconds. Unlike all the previous studies, the first time-resolved two-magnon experiments allowed to claim that the triggered spin dynamics cannot be understood in the frame of classical physics [24,25]. It was reported that the generation of the dynamics of correlations involving pairs of spins in the antiferromagnetic insulator $\mathrm{MnF}_{2}$ induces squeezing. The spin fluctuations in this squeezed state vary periodically in time and are reduced below the level of the ground-state quantum noise. More recently, Bossini et al. reported that the photoexcitation of pairs of magnons with wave vector near the edges of the Brillouin zone, named femto-nanomagnons [23], in antiferromagnetic $\mathrm{KNiF}_{3}$ triggers dynamics of the antiferromagnetic order parameter $\mathbf{L}$. This quantity is defined in terms of the magnetizations of the two magnetic sublattices $\left(\mathbf{M}_{1}, \mathbf{M}_{2}\right) \mathbf{L} \equiv \mathbf{M}_{1}-\mathbf{M}_{2}$ [23]. The generation of pairs of magnons does not simply reduce the magnitude of the order parameter, but it triggers longitudinal oscillations of the modulus of $\mathbf{L}$ at the frequency of the $2 \mathbf{M}$ excitation $\left(2 \omega_{m}\right)$. Despite the highly intriguing results, employing the quantum regime of spin dynamics in photomagnonics is prevented by poor understanding of the fundamental physics of the process. The experimental observations reported in Refs. [23-25] have not found an explanation yet or even contradicted what has been reported before.

First of all, while the detection of two-magnon dynamics in Refs. [24,25] was based on the time-resolved measurement of the transmissivity, Ref. [23] employed time-resolved measurements of the polarization rotation originating from antiferromagnetic linear dichroism. It is not clear if these two detection schemes will give similar results: can the length (a)

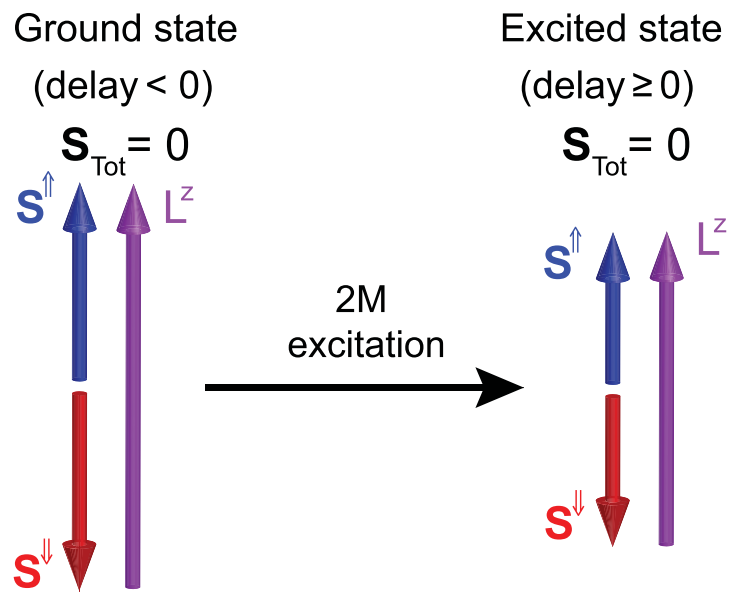

(b)

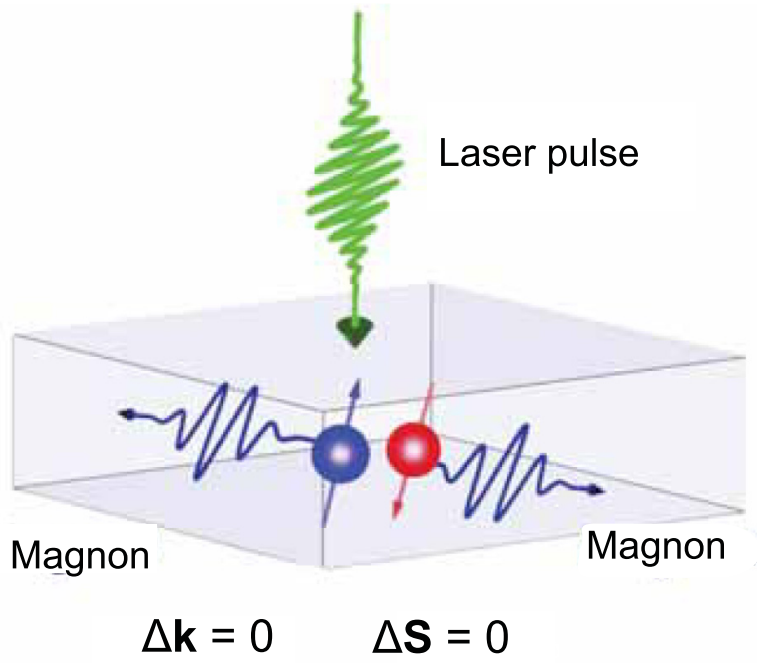

FIG. 1. Conservation laws of the ISRS excitation of the $2 \mathrm{M}$ mode. (a) The total spin is conserved, since magnons are generated by spin flip events occurring in opposite sublattices. (b) The visible excitation light pulse has almost zero wave vector, thus only pairs of magnons with the same and opposite wave vectors can be triggered. As a result, the total wave vector exchanged in the process is zero.

oscillations of $\mathbf{L}$ reported in Ref. [23] be interpreted in terms of the squeezed magnon states from Refs. [24,25] or do Refs. [23-25] report mutually independent phenomena? The magneto-optical experiment [23] demonstrated the possibility to control the phase of the oscillations of the magneto-optical signal varying the polarization of the exciting laser pulse. However, the theory behind this process remains unclear, since it is not established whether the observed modification of the magneto-optical signal depends on a change of sign of the oscillations of $\mathbf{L}$ or on a photoinduced modification of the magneto-optical tensor. Based on the temperature dependence of the efficiencies of the stimulated two-magnon Raman scattering in $\mathrm{FeF}_{2}$, it was suggested [25] that contrary to the spontaneous Raman process long-range spin order is an important, if not essential, component of the coherent two-magnon scattering. It is not clear if this may be a feature specific to $\mathrm{FeF}_{2}$ or a general phenomenon relevant to all antiferromagnets. Aiming to clarify these questions, this work focuses on theoretical and experimental studies of the 
impulsive stimulated Raman scattering and subsequent spin dynamics.

In particular, we show that the description of spin dynamics triggered by the generation of pairs of femto-nanomagnons can be simplified by introducing magnon pair operators. Using coherent states for these operators, we are able to derive an effective macroscopic description beyond the conventional Landau-Lifshitz phenomenology. Moreover, the commonly employed concept of light-induced effective field to describe the photoexcitation of macrospin dynamics $[15,16,26]$ does not apply to the femto-nanomagnons. In our theoretical framework, we formulate an analogous concept, a generalized force responsible for the spin dynamics.

Experimentally the temperature dependence and the pumppolarization dependence of the spin dynamics was explored after impulsive generation of femto-nanomagnons in two antiferromagnets $\mathrm{KNiF}_{3}$ and $\mathrm{K}_{2} \mathrm{NiF}_{4}$ having different magnetocrystalline anisotropy. The microscopic theory does not predict a modification of the spin dynamics if the polarization of the pump beam is changed. In agreement with the theoretical predictions no polarization dependence of the amplitude and phase of the oscillations of the magneto-optical signal was observed in $\mathrm{K}_{2} \mathrm{NiF}_{4}$. However, a dependence was clearly observed in $\mathrm{KNiF}_{3}$. A phenomenological analysis reveals that the perturbations of the spin system induced by different polarization states are not equivalent, although a quantitative description of the experimental observation is still elusive. Using such phenomenological approach, we suggest a possible origin of this phenomenon.

This paper is organized as follows. In Sec. II, the quantum mechanical theory describing the spin dynamics initiated by the generation of femto-nanomagnons is reported. Section III describes the experimental techniques, together with the properties of the materials investigated. Section IV reports the investigation of the temperature dependence of the femto-nanomagnonic dynamics. The experimentally verified criterion allowing to select the proper conditions for the phase control is discussed in Sec. V. The conclusions and perspectives of our work are reported in Sec. VII.

\section{THEORY}

In this section, we present a theoretical description of spin dynamics induced by the two-magnon mode (" $2 \mathrm{M}$ " in the following), meaning with this expression a pair of magnons with the same frequency and same wave vector in magnitude, but opposite in sign. First, we provide an exclusively qualitative discussion of $2 \mathrm{M}$ dynamics, highlighting the qualitative differences between a classical and quantum description. The results of our entire modeling are here summarized and reported without the mathematical formalism, which is then employed in the rest of the section. Second, we introduce a novel microscopic quantum description of $2 \mathrm{M}$ dynamics in terms of boson-pair operators. They allow for a simple analytical treatment, both at zero and at finite temperature. In the third part, we show that using coherent states for the boson-pair operators, we can derive an effectively macroscopic theory for the longitudinal dynamics of the antiferromagnetic vector, which supplements the phenomenological Landau-Lifshitz description for spin dynamics on the fem- tosecond timescale. Moreover, within this macroscopic description we are able to define a generalized force, analogous to the (light-induced) effective magnetic field, commonly employed for long-wavelength magnons. Fourth, we analyze in detail the polarization dependence using a phenomenological treatment of light-matter interaction and we compare this to the results obtained from the quantum model. Finally, we elaborate on various quantum aspects of $2 \mathrm{M}$ dynamics and demonstrate that a natural and unavoidable implication of our theory is that the photoinduced spin dynamics drives entanglement of magnon pairs and, therefore, is a genuine quantum effect.

\section{A. Qualitative description of 2M dynamics}

Two-magnon dynamics has been extensively discussed in the frequency domain, mainly in the context of spontaneous Raman scattering [17-22]. While these theoretical descriptions are essentially quantum, it is not completely clear whether a quantum description is strictly necessary, or arguments in terms of classical spin waves would be adequate as well. Here we are interested in a description of $2 \mathrm{M}$ dynamics in the time domain, triggered by an impulsive stimulated Raman scattering process. To investigate the need for a quantum treatment, we first analyze $2 \mathrm{M}$ dynamics with classical spin wave theory and outline the qualitative features. Second, we show that a qualitatively distinct dynamics arises when quantum correlations between spins at different positions are taken into account. We explain why such quantum features are measurable in macroscopic systems at elevated temperature and elaborate on the excitation mechanism. Finally, we argue that the quantum treatment is required to capture the dynamics observed in the experiments presented in Secs. IV-V.

\section{B. Classical description of two-magnon dynamics and its limitations}

At long wavelengths, the dynamics of magnons in antiferromagnets is conveniently described by the Landau-Lifsthitztype equations of motion for the sublattice magnetizations $\boldsymbol{S}^{\Uparrow}$ and $S^{\Downarrow}$, which are defined as thermodynamic averages of the local spins over physically small volumes (so-called mean field approximation). This classical antiferromagnetic state is usually described in terms of two macroscopic vectors, the magnetization $\mathbf{M}$ and the Néel vector $\mathbf{L}$, the latter is canonically introduced as order parameter for an antiferromagnet [27]:

$$
\mathbf{M}=\frac{N}{2}\left(\boldsymbol{S}^{\Uparrow}+\boldsymbol{S}^{\Downarrow}\right), \quad \mathbf{L}=\frac{N}{2}\left(\boldsymbol{S}^{\Uparrow}-\boldsymbol{S}^{\Downarrow}\right),
$$

where $N$ is the number of magnetic atoms $(N / 2$ per sublattice) per unit volume. These definitions hold for two-sublattice antiferromagnets. For the sake of simplicity, we assume that $\hbar=1$ and that the gyromagnetic ratio equals 1 as well in the definition of both $\mathbf{M}$ and $\mathbf{L}$. Within this classical picture the dynamics of the Néel vector results in the emergence of a small but nonzero magnetization $\mathbf{M} \sim \mathbf{L} \times \partial_{t} \mathbf{L}$ (so-called dynamic magnetization). Hence, both magnetic sublattices are involved in the homogenous oscillation. If the equilibrium orientation of the Néel vector is along the $z$ axis, oscillations of $\mathbf{L}$ at the frequency of antiferromagnetic resonance $\omega_{\mathrm{AFM}}$ 
can be launched by inducing transverse components along the $x$ and $y$ axes. In terms of magnons, the frequency $\omega_{\mathrm{AFM}}$ is the eigenfrequency of spin waves at the center of the Brillouin zone. In other words, the excitation of oscillations of $\mathbf{L}$ corresponds to injection of coherent magnons with approximately zero wave vector. As long as the magnons retain mutual coherence, the modulus of the Néel vector is not reduced, transverse components ( $x$ and $y$ ) oscillate at the frequency $\omega_{\mathrm{AFM}}$ and the $z$-component at the frequency $2 \omega_{\mathrm{AFM}}$. If the magnons were injected via a torque induced by a resonant magnetic-field at the frequency $\omega_{\mathrm{AFM}}$, the amplitude of the oscillations of the transverse components would scale linearly with the magnetic field $\mathrm{H}$. On the other hand, the amplitude of the $2 \omega_{\mathrm{AFM}}$ oscillations of the $z$ component would be proportional to $\mathrm{H}^{2}$, as reported [28]. If the magnons were triggered via ISRS, the amplitude of the transverse and $z$ components would scale linearly and quadratically with respect to the intensity of the pump beam, respectively.

Classical spin wave theory is nevertheless not restricted to homogenous $\mathbf{k} \approx 0$ oscillations. In the framework of an atomistic picture, in which the spin states of individual atoms are disentangled from each other, we consider spin waves with any wave vector in the magnetic Brillouin zone. In particular a magnetic excitation triggered by light, with almost-zero wave vector, can consist of a pair of magnons belonging to different modes, i.e., with wave vectors $\mathbf{k}$ and $-\mathbf{k}$ and with the same frequency. Relevant to our case, for $\mathbf{k}$ close to the Brillouin zone boundary, spin waves are almost localized on one magnetic sublattice. This peculiarity is due to the short wavelength, which is comparable with the lattice constant. Hence, we can envision exciting two distinct spin waves, each one perturbing one of the magnetic sublattices (for simplicity we assume here that the modes are strictly localized, but the argument also holds for eigenmodes that are themselves superpositions of spin waves in each of the sublattices). An illustration of this scenario is given in Fig. 2. Similar to the $\mathbf{k} \approx 0$ case, the local spin oscillations are, to leading order, transverse to the equilibrium value of $\mathbf{L}$, with a welldefined phase relation between spin deflections at different positions (see Fig. 2). Although two spin waves are excited, the oscillation frequency for these transverse deflections is the frequency of each single spin wave mode. Within linear spin wave theory the net change of the longitudinal magnetization is zero, since the change of the local magnetization in each of the magnetic sublattices has opposite sign. On the contrary, the length of the Néel vector is reduced as compared to the equilibrium value. Analogously to the spin waves at the center of the Brillouin zone, also in this case, the second harmonic can appear in the $z$ component as the next-to-the-leadingorder dynamic contribution which scales quadratically with fluence.

\section{Quantum description of $2 \mathrm{M}$ dynamics}

From the aforedescribed analysis it follows that within classical spin wave theory the leading order dynamics is transverse to the equilibrium direction of the Néel vector, while the longitudinal dynamics of the Néel vector occurs at the next-to-the-leading order, at the double frequency of the transverse oscillations and with amplitude scaling

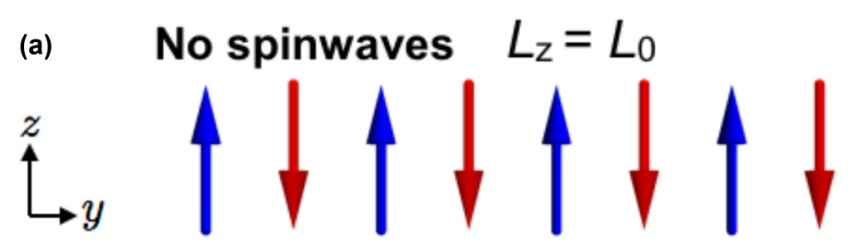

(b) Two spinwaves $L_{z}<L_{0}$
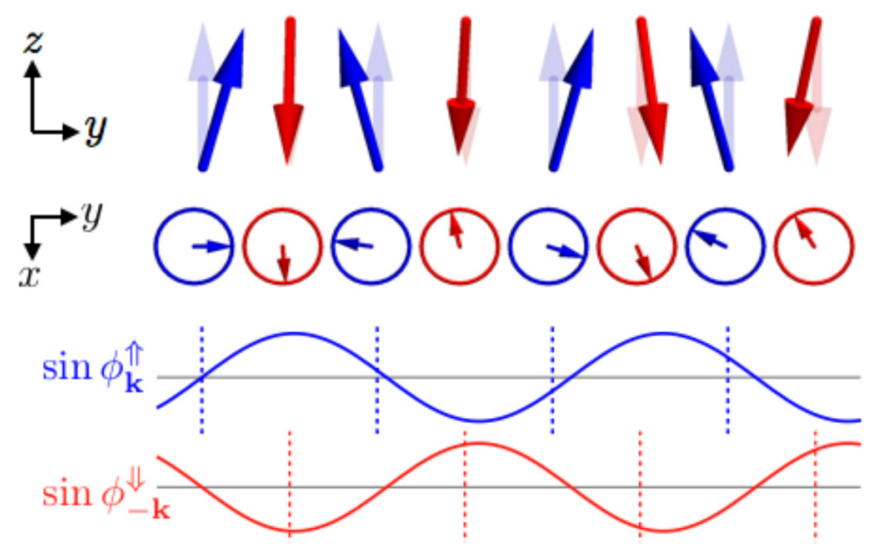

FIG. 2. Illustration of $2 \mathrm{M}$ excitation using classical antiferromagnetic spin waves. (a) Classical Néel state with antiparallel spins at adjacent sites. (b) State with two spin waves, one in each sublattice with opposite wave vector $k= \pm 0.95 \pi / 2 a$ close to the Brillouin zone boundary, where $a$ is the lattice constant. Since in each sublattice the spin is reduced by one unit, the total magnetization is conserved but the Néel vector is reduced. The two bottom panels show the corresponding spin projections in the transverse plane and their phase relationship, with dashed lines indicating lattice points. Spins within the same sublattice are nearly out of phase, while spins in different sublattices are nearly perpendicular in the transverse plane. Therefore the torques induced by neighboring spins cancel almost completely out and the spin waves can be considered localized in one sublattice.

quadratically with the excitation fluence. In the following we will analyze the situation in which quantum correlations between the spin states of the neighboring magnetic atoms cannot be neglected, meaning that we cannot rely on the mean-field approach. For simplicity, we start with a simple example of just two quantum spins with $S=1 / 2$. Quantum correlations $\left\langle\hat{S}_{1} \hat{S}_{2}\right\rangle \neq\left\langle\hat{S}_{1}\right\rangle\left\langle\hat{S}_{2}\right\rangle$ reveal themselves for example when the system is in the superposition state: $|\psi\rangle=$ $c\left|\uparrow_{1}\right\rangle\left|\downarrow_{2}\right\rangle+d\left|\downarrow_{1}\right\rangle\left|\uparrow_{2}\right\rangle$, where the symbols $\uparrow$ and $\downarrow$ indicate two different spin orientations. For this state, the total spin $\left\langle\hat{\mathbf{S}}_{1}+\hat{\mathbf{S}}_{2}\right\rangle=0$, but this does not exclude variation of the individual components $\left\langle\hat{\mathbf{S}}_{1}\right\rangle,\left\langle\hat{\mathbf{S}}_{2}\right\rangle$ (where the brackets mean quantum mechanical average) which means that the length of the Néel vector defined as $|\mathbf{L}| \equiv\left|\left\langle\hat{\mathbf{S}}_{1}-\hat{\mathbf{S}}_{2}\right\rangle\right|=|c|^{2}-|d|^{2}$ can vary. Such changes can be viewed as an elongation of one spin correlated with a shrinking of the other spin, which is accompanied by changes in the spin correlations $\left\langle\hat{S}_{1}^{z} \hat{S}_{2}^{z}\right\rangle=$ $-\left(|c|^{2}+|d|^{2}\right) / 4$ and $\left\langle\hat{S}_{1}^{x} \hat{S}_{2}^{x}\right\rangle=\left\langle\hat{S}_{1}^{y} \hat{S}_{2}^{y}\right\rangle=\left(c d^{*}+c^{*} d\right) / 4$.

In a more general picture of an antiferromagnet with $N$ correlated magnetic atoms, the magnetization and the Néel vector are defined through both quantum-mechanical average 


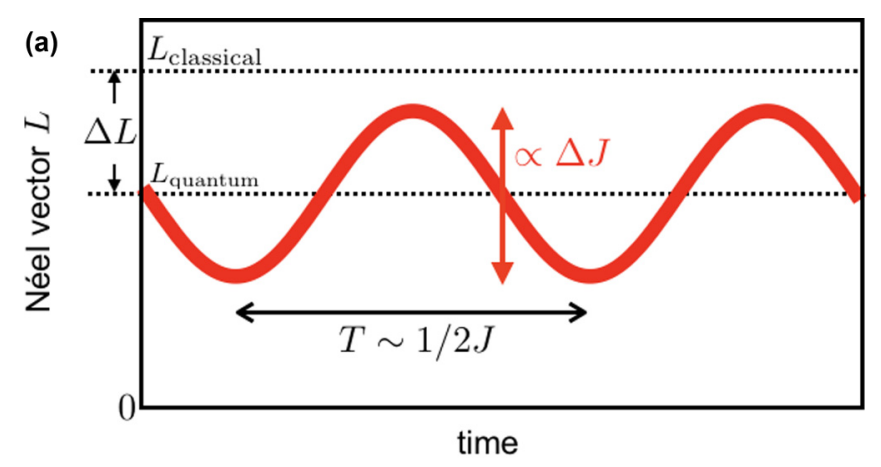

(b)

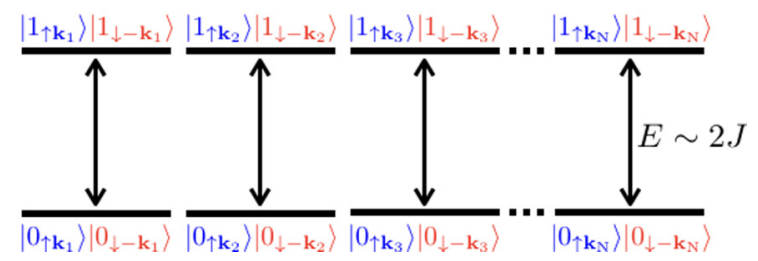

FIG. 3. Illustration of the oscillation of the Néel vector. (a) In a quantum antiferromagnet, the magnitude of the Néel vector $L=$ $|\mathbf{L}|$ is reduced with respect to the classical value by an amount $\Delta L$ (dashed lines). This reduction originates from dressing with two-magnon excitations. An ultrafast perturbation of the exchange interaction $(\Delta J)$ changes the contribution of these dressed states and triggers longitudinal oscillations. (b) The emergence of such oscillations can be understood from the coherent excitation of an ensemble of two-level systems. Each of these two-level systems represents coherent oscillations between the Néel state $\left|0_{\uparrow \mathbf{k}_{i}}\right\rangle\left|0_{\downarrow-\mathbf{k}_{i}}\right\rangle$ and a state with two magnons excited $\left|1_{\uparrow \mathbf{k}_{i}}\right\rangle\left|1_{\downarrow-\mathbf{k}_{i}}\right\rangle$, separated by an energy $E \sim 2 J$.

and average in real space ( $i$ and $j$ are indices for lattice sites):

$$
\begin{aligned}
\mathbf{M} & :=\left(\sum_{i}\left\langle\hat{\mathbf{S}}_{i}^{\Uparrow}\right\rangle+\sum_{j}\left\langle\hat{\mathbf{S}}_{j}^{\Downarrow}\right\rangle\right), \\
\mathbf{L} & :=\left(\sum_{i}\left\langle\hat{\mathbf{S}}_{i}^{\Uparrow}\right\rangle-\sum_{j}\left\langle\hat{\mathbf{S}}_{j}^{\Downarrow}\right\rangle\right) .
\end{aligned}
$$

and the sums are considered in the unit volume. In the limit of vanishingly small correlations between neighboring spins, definition (2) coincides with the classical vectors Eq. (1).

In such extended systems, quantum correlations reveal themselves in a coherent dynamics which can be described as quantum Rabi-like oscillations between the ground Néel state and the excited state (i.e., 2M state), both of which are represented in Fig. 2. Since the magnitude of $\mathbf{L}$ is reduced in the state with $2 \mathrm{M}$ as compared to the Néel state, a time-dependent superposition of these two states gives rise to longitudinal oscillations of $\mathbf{L}$, already within the harmonic magnon theory. Hence, we can understand the $2 \mathrm{M}$ dynamics relying on a simplified picture of a two-level system, in which coherent quantum oscillations occur between two-particle states: the Néel state, which can be expressed in terms of absence of any magnons i.e., $\left|0_{\uparrow \mathbf{k}}\right\rangle\left|0_{\downarrow-\mathbf{k}}\right\rangle$, and a state in which the $2 \mathrm{M}$ mode is excited $\left|1_{\uparrow \mathbf{k}}\right\rangle\left|1_{\downarrow-\mathbf{k}}\right\rangle$ (see Fig. 3). An extended antiferromagnetic systems can be envisaged as a large ensemble of such two-level systems, one for each $\mathbf{k}$ value. A short optical excitation pulse in the ISRS scheme triggers oscillations with the same initial phase for each two-level system. As long as the two-level systems remain mutually coherent, the length of the $\mathbf{L}$ vector oscillates with a frequency twice bigger than the coherent magnons. Although the spectrum of magnons in a bulk antiferromagnet is broad, the overwhelming contribution to the magnon density of states originates from magnons with wave vector close to the Brillouin zone boundary, $\mathbf{k} \approx \mathbf{k}_{\mathrm{R}}=$ $[ \pm \pi / 2 a, \pm \pi / 2 a, \pm \pi / 2 a]$, where $a$ is the lattice constant. For such magnons, the energy of a single quantum is $\hbar \omega_{\mathrm{R}} \approx z S J$, where $z$ is the number of nearest neighbor spins with spin $S$ and $J$ is the parameter of the exchange interaction. It means that as long as magnons with energy $\hbar \omega_{\mathrm{R}}$ and opposite $\mathbf{k}$ remain coherent, the Néel vector oscillates at the frequency $2 \omega_{\mathrm{R}}$. Hence, within the quantum description the double frequency is already predicted within the harmonic magnon theory. This is the main difference between the quantum and classical descriptions. In particular, if the Néel vector is aligned along the $z$ axis and pairs of coherent magnons with equal frequencies and opposite wave vectors are photoinduced via ISRS, the quantum theory predicts oscillations of the length of the antiferromagnetic vector at a frequency which is twice the frequency of the individual magnons. The amplitude of the oscillations will scale linearly with the pump fluence. In the classical theory, the length of the Néel vector does not oscillate in a linear regime, although oscillations of the $z$ component of the Néel vector can be achieved, in which case the amplitude of the oscillations scales quadratically with the pump fluence. In Sec. II E below, we focus exclusively on a quantum description of $2 \mathrm{M}$ dynamics.

\section{Excitation mechanism}

Next we elaborate on the excitation mechanism in the quantum mechanical framework. For an individual two-level system, the energy splitting is given by $E=2 \hbar \omega_{\mathrm{R}}=2 z J S$. Although a classical antiferromagnet can be described by the Néel state, this is not an eigenstate of the Heisenberg Hamiltonian. Therefore the ground state of a quantum antiferromagnet must be different; in particular it is given by the superposition of the Néel state and states in which two correlated spin flips and thus magnons are excited. The correlated spin flips are induced by the canonical ladder operators $\hat{S}_{i}^{+} \hat{S}_{j}^{-}$appearing in the Heisenberg Hamiltonian and represent transitions ... $|\uparrow\rangle|\downarrow\rangle|\uparrow\rangle|\downarrow\rangle \cdots \rightarrow \cdots|\uparrow\rangle|\uparrow\rangle|\downarrow\rangle|\downarrow\rangle \cdots$. This causes a ground state in which the Néel vector is reduced with respect to the classical value, as illustrated by dashed lines in Fig. 3. These fluctuations, which are a purely quantum mechanical effects, are not thermal and thus are present even at zero temperature. Since the fluctuations are dominated by longwavelength low-energy magnons, they are suppressed as the temperature increases. Moreover, no phase relation exists among the magnons generated in this process.

The initial state thus has a nonzero population of the magnon states relevant for the $2 \mathrm{M}$ mode. Thus a sudden perturbation of the exchange interaction $\Delta J$ is sufficient to induce coherent oscillations of the population between the ground state and the excited $2 \mathrm{M}$ state. The longitudinal component of $\mathbf{L}$ is hence further reduced. These oscillations cannot be quenched by thermal fluctuations since the energy 
splitting $E$ is large compared to the thermal energy, even at room temperature. In particular, $E>k_{\mathrm{B}} T_{\mathrm{N}}$, where $T_{\mathrm{N}}$ is the Néel temperature. We also note that perturbations $\Delta J$ in classical spin systems at finite temperature give rise only to a rapid relaxation, not coherent oscillations [29]. Moreover, the oscillations show a macroscopic well-defined phase, since the excitation is impulsive: therefore a macroscopic ensemble measurement can reveal them. In the quantum mechanical scenario, we are depicting, the initial phase of the oscillations is determined both by the sign of the $\Delta J$ and the polarization of the optical pump pulse. Although an optical perturbation of the exchange interactions induces in principle a change $\Delta J$ homogenous throughout the system; the pump pulse perturbs the exchange bonds along different crystallographic directions in a nonequivalent way, depending on the direction of the electric field of light (i.e., polarization). The light-matter interaction can also depend on the orientation of the electrical field with respect to the equilibrium orientation of the Néel vector. Thus oscillations induced by pump pulses parallel or perpendicular to the Néel vector can have different phases, although in general it is expected that the contribution of the perturbation of exchange interaction dominates. This is because spin-orbit interactions are much weaker than the exchange interactions and perturbation of the spin-orbit coupling alone cannot trigger the purely longitudinal oscillation of the Néel vector.

\section{E. Microscopic theory of two-magnon dynamics}

In this section, we present a more mathematical treatment of the microscopic quantum description of impulsively stimulated $2 \mathrm{M}$ dynamics, similarly to what has been already introduced in the literature [23-25] and as outlined qualitatively in the previous section. We give a self-contained derivation starting with the perturbation of exchange interactions as the excitation mechanism. Subsequently and beyond the existing theory, we show how the theoretical solution can be simplified by the introduction of Bose pair operators which allow us to link the dynamics of the order parameter with the dynamics of the spin correlations. Such a connection was previously shown only at zero temperature.

\section{Effective Hamiltonian of light-matter interaction}

In the literature, $\mathrm{K}_{2} \mathrm{NiF}_{4}$ and $\mathrm{KNiF}_{3}$ are considered as prototype Heisenberg quantum antiferromagnets on simple cubic lattice structures in two and three dimensions, respectively [30]. Therefore, we describe the microscopic excitation mechanism of the ISRS on the $2 \mathrm{M}$ mode considering the quantum Heisenberg model with only nearest neighbor exchange interactions parametrized with the constant $J>0$ :

$$
H_{0}=J \sum_{i, \delta} \hat{\mathbf{S}}_{i} \cdot \hat{\mathbf{S}}_{i+\delta}
$$

where $\hat{\mathbf{S}}_{i}$ is the spin operator at site $i$ and $\delta$ is a vector connecting a magnetic site with one of its nearest neighbors on the opposite sublattice. Note that the modulus of $\delta$ is equal to the lattice constant $a$ [see Fig. 4(a)]. In general, the Raman tensor responsible for $2 \mathrm{M}$ scattering can be derived from a symmetry analysis and it represents a light-induced modification of the exchange interaction. [17,24,31]. Here, to facilitate a simple

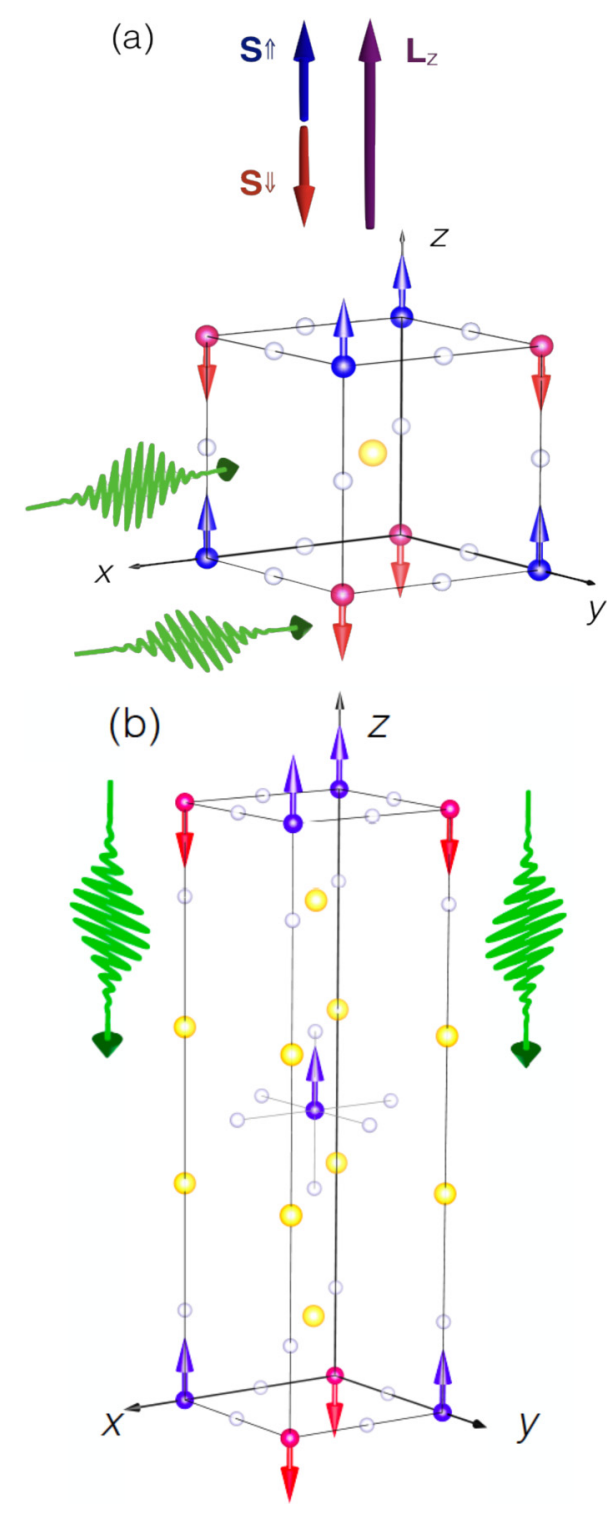

FIG. 4. Crystallographic and magnetic structures of $\mathrm{KNiF}_{3}$ (a) and $\mathrm{K}_{2} \mathrm{NiF}_{4}$ (b). Note that the isolated spin between the $\mathrm{NiF}_{2}$ layers in $\mathrm{K}_{2} \mathrm{NiF}_{4}$ is not relevant for the magnetic structure of this compound (see main text). We can thus consider these two samples as a $3 \mathrm{D}$ and a $2 \mathrm{D}$ Heisenberg antiferromagnets, respectively. The only exchange interaction relevant to the present study is between nearest-neighbor spins, which are located on two different ionic sites and belong to oppositely oriented magnetic sublattices. The total spins of each sublattice, $\mathbf{S}^{\Uparrow}$ and $\mathbf{S}^{\Downarrow}$, are represented: they are obtained by summing all the magnetic moments belonging to the $\Uparrow$ and $\Downarrow$ sublattices, respectively. The magnetization (M) and the antiferromagnetic vector $(\mathbf{L})$ are defined: while the former vanishes, the latter is the order parameter of an antiferromagnet. The propagation directions and the polarizations of the pump pulses employed during the experiments are represented by the green pulses in the figure.

microscopic description of $2 \mathrm{M}$ excitation, we discuss lightinduced perturbations to the exchange interaction as derived from the electronic Hubbard model [32-34]. For a given bond along $\boldsymbol{\delta}$, we have

$$
\Delta J(\boldsymbol{\delta})=\frac{t_{0}^{2}}{2 U} \frac{\left(e \boldsymbol{\delta} \cdot \mathbf{E}_{0}\right)^{2}}{U^{2}-\hbar^{2} \omega^{2}}
$$


where $t_{0}$ is the hopping integral, $U$ the onsite Coulomb interaction, $e$ the unit charge and $\hbar=h / 2 \pi$ with $h$ being the Planck constant. The symbol $\omega$ represents the angular frequency of the electric field of light, while $\delta \cdot \mathbf{E}_{0} / a$ is the projection of the optical electric field along the nearest-neighbor bond between two spins. This equation reveals how $J$ can be changed by an off-resonant driving of the charge-transfer transition in the Hubbard model. Hence, this approach takes into account the virtual charge-transfer processes between sites belonging to the same band, but not the electric dipole transitions to higher bands. Nevertheless, already from the current model, we observe that the sign of $\Delta J$ is different for off-resonant driving laser pulses with photon energy tuned below and above the charge-transfer gap. The experiments here reported always employed a driving electric field oscillating at frequencies below the charge-transfer gap and therefore no change of sign of $\Delta J$ is expected from the model. Extending the model, by including more bands and provided that the symmetry of the crystal allows it, the combination of all transitions can cause the sign of $\Delta J$ to become dependent on the orientation of the electric field of light with respect to the Néel vector and the crystallographic axes (see the phenomenological analysis in Sec. II H).

Considering exclusively optical perturbations to the exchange, the light matter interaction takes the form

$$
\delta H=\frac{1}{2} f(t) \sum_{i, \delta} \Delta J(\delta) \hat{\mathbf{S}}_{i} \cdot \hat{\mathbf{S}}_{i+\delta},
$$

where the function $f(t)$, which is normalized to 1 at its maximum value, describes the time profile of the light pulse.

\section{Magnon modes}

The strength required to modify $J$ by an amount comparable with the exchange itself is of the same order as the atomic electric field. As the intensity of the pump signal is smaller than the atomic electric field, we assume that the photoinduced fluctuations are small deviations from the equilibrium state $(\Delta J \ll J)$. Such fluctuations can be described in terms of magnon modes. Following the standard approach [35], we introduce bosonic annihilation (creation) operators $\hat{\alpha}_{\mathbf{k}}\left(\hat{\alpha}_{\mathbf{k}}^{\dagger}\right)$, $\hat{\beta}_{\mathbf{k}}\left(\hat{\beta}_{\mathbf{k}}^{\dagger}\right)$, which correspond to two types of magnon modes and whose detailed derivation is given in Appendix A. As aforementioned, the structure of the eigenmodes strongly depends upon the $\mathbf{k}$ vector. In particular, for $\mathbf{k} \approx \mathbf{k}_{\mathrm{R}}$ operator $\hat{\alpha}_{\mathbf{k}}^{\dagger}$ $\left(\hat{\beta}_{\mathbf{k}}^{\dagger}\right)$ creates spin excitations mainly in one magnetic sublattice A (B).

To represent the wave function of the magnon modes we use the basis of the Fock states with a fixed number $n_{\uparrow \mathbf{k}}$ and $n_{\downarrow-\mathbf{k}}$ in each mode, where the arrows indicate the two subalattices (to which the magnons belong) and $\mathbf{k}$ is the wave vector. The one-magnon operators act on the Fock states in a standard way:

$$
\begin{aligned}
\hat{\alpha}_{\mathbf{k}}^{\dagger}\left|n_{\uparrow \mathbf{k}}\right\rangle & =\sqrt{n_{\uparrow \mathbf{k}}+1}\left|n_{\uparrow \mathbf{k}}+1\right\rangle, \\
\hat{\beta}_{-\mathbf{k}}^{\dagger}\left|n_{\downarrow-\mathbf{k}}\right\rangle & =\sqrt{n_{\downarrow-\mathbf{k}}+1}\left|n_{\downarrow-\mathbf{k}}+1\right\rangle,
\end{aligned}
$$

and the vacuum states are $\hat{\alpha}_{\mathbf{k}}\left|0_{\uparrow \mathbf{k}}\right\rangle=0, \hat{\beta}_{-\mathbf{k}}\left|0_{\downarrow-\mathbf{k}}\right\rangle=0$.
Neglecting magnon-magnon interactions, the Hamiltonians in Eqs. (3) and (5) are expressed as follows:

$$
\begin{aligned}
\hat{H}_{0}= & E_{0}+\sum_{\mathbf{k}} \hbar \omega_{\mathbf{k}}\left[\hat{\alpha}_{\mathbf{k}}^{\dagger} \hat{\alpha}_{\mathbf{k}}+\hat{\beta}_{\mathbf{k}}^{\dagger} \hat{\beta}_{\mathbf{k}}+1\right], \\
\delta \hat{H}= & f(t) \sum_{\mathbf{k}}\left\{\hbar \delta \omega_{\mathbf{k}}\left[\hat{\alpha}_{\mathbf{k}}^{\dagger} \hat{\alpha}_{\mathbf{k}}+\hat{\beta}_{\mathbf{k}}^{\dagger} \hat{\beta}_{\mathbf{k}}+1\right]\right. \\
& \left.+\hbar V_{\mathbf{k}}\left[\hat{\alpha}_{\mathbf{k}} \hat{\beta}_{-\mathbf{k}}+\hat{\alpha}_{\mathbf{k}}^{\dagger} \hat{\beta}_{-\mathbf{k}}^{\dagger}\right]\right\},
\end{aligned}
$$

where the constant $E_{0}=\frac{\hbar}{2}\left(\Omega+\delta \omega_{\mathrm{R}}\right) N(S+1)$ sets the reference level energy, $\Omega \equiv z_{N} J S / \hbar\left(z_{N}\right.$ being the number of nearest neighbors) and $\delta \omega_{\mathrm{R}} \equiv z_{N} \Delta J S / \hbar$ are the magnon frequency and light-matter coupling constant at the $R$ point, respectively. We observe that while $\hat{H}_{0}$ is diagonal in the magnon operators, $\delta \hat{H}$ contains also an off-diagonal term. The term containing $V_{\mathbf{k}}$ is responsible for the excitation and annihilation of magnon pairs during the action of the pump pulse. The perturbation of the exchange interaction by the optical stimulus induces also an effective shift of the magnon frequency amounting to $\delta \omega_{\mathrm{R}}$, which is limited to the duration of the pump laser pulse. Hence, the $\delta \omega_{\mathrm{R}}$ should not be interpreted as a modification of the frequency of the magnons observed in our experimental data at positive delays, but only as an expression in the light-spin interaction. The parameters in Eqs. (7) and (8) are defined as

$$
\begin{gathered}
\omega_{\mathbf{k}}=\Omega \sqrt{1-\gamma_{\mathbf{k}}^{2}}, \\
\delta \omega_{\mathbf{k}}=\delta \omega_{\mathrm{R}} \frac{1-\xi_{\mathbf{k}} \gamma_{\mathbf{k}}}{\sqrt{1-\gamma_{\mathbf{k}}^{2}}} \\
V_{\mathbf{k}}=V_{\mathbf{k}}^{*}=\delta \omega_{\mathrm{R}} \frac{\xi_{\mathbf{k}}-\gamma_{\mathbf{k}}}{\sqrt{1-\gamma_{\mathbf{k}}^{2}}} .
\end{gathered}
$$

Here the factors $\gamma_{\mathbf{k}}=\frac{1}{z} \sum_{\delta} \exp (i \mathbf{k} \cdot \boldsymbol{\delta})$ and $\xi_{\mathbf{k}}=\frac{1}{z a^{2}} \sum_{\delta}(\hat{e}$. $\hat{\boldsymbol{\delta}})^{2} \exp (i \mathbf{k} \cdot \boldsymbol{\delta})$ depend on the structure of the lattice and on the orientation of the electric field. For a cubic lattice $\left(\mathrm{KNiF}_{3}\right)$, which is relevant for the experiments here discussed, it follows that

$$
\gamma_{\mathbf{k}}=\frac{1}{3} \sum_{j=x, y, z} \cos \left(k_{j} a\right), \quad \xi_{\mathbf{k}}=\frac{1}{3} \sum_{j=x, y, z} e_{j}^{2} \cos \left(k_{j} a\right) .
$$

In a tetragonal $\left(\mathrm{K}_{2} \mathrm{NiF}_{4}\right)$ system, we have

$$
\gamma_{\mathbf{k}}=\frac{1}{2} \sum_{j=x, y} \cos \left(k_{j} a\right), \quad \xi_{\mathbf{k}}=\frac{1}{2} \sum_{j=x, y} e_{j}^{2} \cos \left(k_{j} a\right) .
$$

\section{F. 2M operators}

To solve the spin dynamics triggered by the light-matter interaction described by Eq. (8), it is convenient to introduce operators that directly work on magnon pairs. We define them as

$$
\begin{aligned}
& \hat{K}_{\mathbf{k}}^{z}=\left(\hat{\alpha}_{\mathbf{k}}^{\dagger} \hat{\alpha}_{\mathbf{k}}+\hat{\beta}_{-\mathbf{k}}^{\dagger} \hat{\beta}_{-\mathbf{k}}+1\right) / 2, \\
& \hat{K}_{\mathbf{k}}^{+}=\hat{\alpha}_{\mathbf{k}}^{\dagger} \hat{\beta}_{-\mathbf{k}}^{\dagger}, \quad \hat{K}_{\mathbf{k}}^{-}=\hat{\alpha}_{\mathbf{k}} \hat{\beta}_{-\mathbf{k}},
\end{aligned}
$$

where $z$ is the quantization axis which coincides with the equilibrium orientation of spins. The physical interpretation of these operators is that $\hat{K}_{\mathbf{k}}^{z}$ is the number operator in the 
two-magnon mode basis, while $\hat{K}_{\mathbf{k}}^{+}, \hat{K}_{\mathbf{k}}^{-}$describe creation and annihilation of $2 \mathrm{M}$ mode states, respectively. In the context of a coherent state description, such magnon-pair operators are also known as Perelomov operators [36,37], while in the theory of magnetism they are referred to as hyperbolic operators. ${ }^{1}$ As it follows from the Bose commutator relations $\left[\alpha_{\mathbf{k}}, \alpha_{\mathbf{k}}^{\dagger}\right]=1$ and $\left[\beta_{\mathbf{k}}, \beta_{\mathbf{k}}^{\dagger}\right]=1$, the magnon-pair operators exhibit simple commutation relations:

$$
\left[\hat{K}_{\mathbf{k}}^{z}, \hat{K}_{\mathbf{k}}^{ \pm}\right]= \pm \hat{K}_{\mathbf{k}}^{ \pm}, \quad\left[\hat{K}_{\mathbf{k}}^{-}, \hat{K}_{\mathbf{k}}^{+}\right]=2 \hat{K}_{\mathbf{k}}^{z} .
$$

We also note that the magnon-pair operators are distinct from Schwinger bosons. While both deal with introduction of two types of bosons, the bosons introduced by Schwinger are introduced for each single spin or one single mode, with an additional constraint to satisfy spin conservation, i.e., $\mathrm{SU}(2)$ symmetry. Instead, the magnon-pair operators combine two bosons that correspond to two different magnon modes. The role of spin conservation is played by the so-called Casimir invariant

$$
\begin{aligned}
\hat{Q}_{\mathbf{k}} & =\frac{1}{2}\left(\hat{K}_{\mathbf{k}}^{+} \hat{K}_{\mathbf{k}}^{-}+\hat{K}_{\mathbf{k}}^{-} \hat{K}_{\mathbf{k}}^{+}\right)-\left(\hat{K}_{\mathbf{k}}^{z}\right)^{2} \\
& =\frac{1}{4}\left[1-\left(\hat{\alpha}_{\mathbf{k}}^{\dagger} \hat{\alpha}_{\mathbf{k}}-\hat{\beta}_{-\mathbf{k}}^{\dagger} \hat{\beta}_{-\mathbf{k}}\right)^{2}\right],
\end{aligned}
$$

which commutes with all $\hat{K}$ operators. In particular, the conservation law $\Delta S=0$ is respected by the magnon pair operators, since although the total number of magnons $n_{\uparrow \mathbf{k}}+$ $n_{\downarrow-\mathbf{k}}$ can be changed, terms with $\hat{K}_{\mathbf{k}}^{ \pm}$change the number of magnons in each of the sublattices by the same amount $\left(n_{\uparrow \mathbf{k}}=n_{\downarrow-\mathbf{k}}\right)$. In addition, only pairs of magnons of opposite $\mathbf{k}$ are excited, respecting $\Delta \mathbf{k}=0$. In the $2 \mathrm{M}$ basis, we can express the Casimir invariant as $\hat{Q}_{\mathbf{k}}=\left[1-\left(n_{\uparrow \mathbf{k}}-n_{\downarrow-\mathbf{k}}\right)^{2}\right] / 4$. Mathematically, this conservation law is reflected by the fact that the commutation relations differ from spin commutation relations and generate the Lie algebra of $\mathrm{SU}(1,1)$ instead of $\mathrm{SU}(2)$ [36]. In other words, while spin operators describe rotations constrained to the unit sphere, the operators $\hat{K}$ describe abstract rotations constrained to the hyperbolic unit sphere. As we will see, physically this corresponds to longitudinal oscillations that conserve the total spin instead of precessions that keep the magnitude of the spins conserved.

As a result, to work with $\hat{K}$ operators we can choose a subspace of the Hilbert space spanned by the vectors $\left|n_{\uparrow \mathbf{k}}\right\rangle\left|n_{\downarrow-\mathbf{k}}\right\rangle=\left|n_{\mathbf{k}}\right\rangle\left|n_{\mathbf{k}}\right\rangle$. These $2 \mathrm{M}$ states correspond to the discrete representation of the group $\mathrm{SU}(1,1)$ for which the Casimir invariant $\hat{Q}=1 / 4$. The operators $\hat{K}_{\mathbf{k}}^{+}\left(\hat{K}_{\mathbf{k}}^{-}\right)$create (annihilate) the two-magnon states:

$$
\begin{aligned}
\hat{K}_{\mathbf{k}}^{+}\left|n_{\mathbf{k}}\right\rangle\left|n_{\mathbf{k}}\right\rangle & =\left(n_{\mathbf{k}}+1\right)\left|n_{\mathbf{k}}+1\right\rangle\left|n_{\mathbf{k}}+1\right\rangle, \\
\hat{K}_{\mathbf{k}}^{-}\left|n_{\mathbf{k}}\right\rangle\left|n_{\mathbf{k}}\right\rangle & =n_{\mathbf{k}}\left|n_{\mathbf{k}}-1\right\rangle\left|n_{\mathbf{k}}-1\right\rangle,
\end{aligned}
$$

and these states are the eigenstates of the operator $\hat{K}_{\mathbf{k}}^{z}$ :

$$
\hat{K}_{\mathbf{k}}^{z}\left|n_{\mathbf{k}}\right\rangle\left|n_{\mathbf{k}}\right\rangle=\left(n_{\mathbf{k}}+1 / 2\right)\left|n_{\mathbf{k}}\right\rangle\left|n_{\mathbf{k}}\right\rangle .
$$

\footnotetext{
${ }^{1}$ To the best of our knowledge, these magnon-pair operators have not been introduced before in the context of antiferromagnetic magnon theory.
}

The vacuum states for the magnon-pair operators are defined as follows:

$$
\hat{K}_{\mathbf{k}}^{-}\left|0_{\uparrow \mathbf{k}}\right\rangle\left|0_{\downarrow-\mathbf{k}}\right\rangle=0 .
$$

Substituting Eqs. (14) and (15) in the Hamiltonians (3) and (5). we obtain

$$
\begin{gathered}
\hat{H}_{0}=E_{0}+\sum_{\mathbf{k}} 2 \hbar \omega_{\mathbf{k}} \hat{K}_{\mathbf{k}}^{z}, \\
\delta \hat{H}=f(t) \sum_{\mathbf{k}}\left[2 \hbar \delta \omega_{\mathbf{k}} \hat{K}_{\mathbf{k}}^{z}+\hbar V_{\mathbf{k}}\left(\hat{K}_{\mathbf{k}}^{+}+\hat{K}_{\mathbf{k}}^{-}\right)\right] .
\end{gathered}
$$

In the next section, we use the effective Hamiltonians (21) and (22) for the analysis of light-induced spin dynamics.

\section{Spin-spin correlations and the Néel vector}

The main difference between classical and quantum dynamics arises due to the nonlocal quantum spin-spin correlations that are neglected in the conventional classical description. A direct link between the longitudinal component of the Néel vector and the longitudinal correlators can be obtained within harmonic magnon theory. From definition (2), we obtain (see Appendix B)

$$
\hat{L}_{z}=\frac{N S}{2}-\frac{1}{z_{N} S} \sum_{j, \delta} \hat{S}_{j}^{z} \hat{S}_{j+\delta}^{z},
$$

in accordance with what was previously derived [23].

The individual components of the spin correlations can be then expressed in terms of the magnon-pair operators. As detailed in Appendix A, we obtain for the longitudinal spin correlator along the quantization axis the following result:

$$
\begin{aligned}
\sum_{j, \delta} \hat{S}_{j}^{z} \hat{S}_{j+\delta}^{z}= & -\frac{1}{2} z_{N} N S^{2}+z_{N} S \sum_{\mathbf{k}}\left(\frac{2 \hat{K}_{\mathbf{k}}^{z}}{\sqrt{1-\gamma_{\mathbf{k}}^{2}}}-1\right) \\
& -z_{N} S \sum_{\mathbf{k}} \frac{\gamma_{\mathbf{k}}}{\sqrt{1-\gamma_{\mathbf{k}}^{2}}}\left[\hat{K}_{\mathbf{k}}^{+}+\hat{K}_{\mathbf{k}}^{-}\right] .
\end{aligned}
$$

Equation (24) shows that the longitudinal spin correlations and hence, $\hat{L}_{z}$ includes operators $\hat{K}_{\mathbf{k}}^{ \pm}$which do not commute with $\hat{H}_{0}$. In other words, both longitudinal correlators and the Néel vector are not conserved quantities and hence can show dynamics even if the total energy is conserved.

Instead, the longitudinal component of the magnetization, like the $\hat{Q}_{\mathbf{k}}$ operator, depends on the difference between the number of $\uparrow$ and $\downarrow$ magnons,

$$
\hat{M}_{z}=\sum_{\mathbf{k}}\left(\hat{\alpha}_{\mathbf{k}}^{\dagger} \hat{\alpha}_{\mathbf{k}}-\hat{\beta}_{-\mathbf{k}}^{\dagger} \hat{\beta}_{-\mathbf{k}}\right) .
$$

We thus find that $\left[M_{z}, \hat{Q}_{\mathbf{k}}\right]=0$ and in the case when only $2 \mathrm{M}$ Raman processes are considered, $M_{z}=0$ during the whole dynamics.

It should be noted that the transverse spin components, which would give rise to a magnetization $M_{x, y}$ detectable via Faraday rotation, are expressed through linear combinations of one-magnon mode operators $\hat{\alpha}_{\mathbf{k}}, \hat{\beta}_{\mathbf{k}}$. Here and below, we restrict our theoretical model to $2 \mathrm{M}$ Raman processes, $L_{x, y}=$ $M_{x, y}=0$, in accordance with the experimentally demonstrated absence of the Faraday rotation [23]. 


\section{Impulsively stimulated spin dynamics}

The introduced magnon-pair operators allow to model properly the spin dynamics generated by the $2 \mathrm{M}$ mode. In particular, we show that we can describe the dynamics without knowledge of the full initial state, which makes our results applicable both at zero temperature and at finite temperature. To this end, it is convenient to employ the interaction picture and to introduce the time-dependent operators $\hat{K}(t)=\exp \left(i \hat{H}_{0} t / \hbar\right) \hat{K} \exp \left(-i \hat{H}_{0} t / \hbar\right)$. Using the commutation relations (16), we obtain

$$
\hat{K}_{\mathbf{k}}^{ \pm}(t)=\hat{K}_{\mathbf{k}}^{ \pm} e^{ \pm \mathrm{i} 2 \omega_{\mathbf{k}} t}, \quad \hat{K}_{\mathbf{k}}^{z}(t)=\hat{K}_{\mathbf{k}}^{z} .
$$

Note that the operator $\hat{K}_{\mathbf{k}}^{z}(t)$, which defines the number of magnons, commutes with $\hat{H}_{0}$ and hence its expectation value is time-independent. The time dependence of a quantum mechanical state is then defined by the unitary evolution operator $\hat{U}(t, 0)$ which satisfies the equation

$i \hbar \frac{d \hat{U}(t, 0)}{d t}=f(t) \sum_{\mathbf{k}}\left[2 \hbar \delta \omega_{\mathbf{k}} \hat{K}_{\mathbf{k}}^{z}+\hbar V_{\mathbf{k}}\left(\hat{K}_{\mathbf{k}}^{+}+\hat{K}_{\mathbf{k}}^{-}\right)\right] \hat{U}(t, 0)$.

As in the experiments the duration of the laser pulses $\tau_{\text {pulse }}$ is considerably smaller than the oscillation period of the magnons: $\tau_{\text {pulse }} \Omega \ll 1$. So we model the temporal profile of the pump pulses as $f(t)=\tau_{\text {pulse }} \delta(t)$, where $\delta$ represents the Dirac delta function. Exploiting that in the harmonic approximation the Hamiltonian is diagonal in $\mathbf{k}$, the time-evolution operator can be calculated explicitly as follows:

$$
\begin{aligned}
\hat{U}(t, 0) & =\prod_{\mathbf{k}} \hat{U}_{\mathbf{k}}(t, 0) \\
\hat{U}_{\mathbf{k}}(t, 0) & =\exp \left\{i \tau_{\text {pulse }}\left[V_{\mathbf{k}}\left(\hat{K}_{\mathbf{k}}^{+}(t)+\hat{K}_{\mathbf{k}}^{-}(t)\right)+\delta \omega_{\mathbf{k}} \hat{K}_{\mathbf{k}}^{z}(t)\right]\right\} .
\end{aligned}
$$

The temporal evolution of the expectation value of an operator $\hat{K}_{\mathbf{k}}$ is then calculated as $K_{\mathbf{k}}(t)=\left\langle\hat{U}_{\mathbf{k}}^{\dagger}(t, 0) \hat{K}_{\mathbf{k}} \hat{U}_{\mathbf{k}}(t, 0)\right\rangle$, where the symbol $\langle\ldots\rangle$ means quantum-mechanical averaging over the initial state. Using the commutation relations in Eq. (16), we obtain the following expressions for the observables $K_{\mathbf{k}}(t)$ :

$$
\begin{aligned}
K_{\mathbf{k}}^{+}(t)+K_{\mathbf{k}}^{-}(t)= & -4 V_{\mathbf{k}} \tau_{\text {pulse }}\left\langle\hat{K}_{\mathbf{k}}^{z}\right\rangle \sin \left(2 \omega_{\mathbf{k}} t-\varphi_{\mathbf{k}}\right) \\
& +\left\langle\hat{K}_{\mathbf{k}}^{+}\right\rangle e^{2 i \varphi_{\mathbf{k}}}+\left\langle\hat{K}_{\mathbf{k}}^{-}\right\rangle e^{-2 i \varphi_{\mathbf{k}}}, \\
K_{\mathbf{k}}^{z}(t)= & \left\langle\hat{K}_{\mathbf{k}}^{z}\right\rangle-2 V_{\mathbf{k}} \tau_{\text {pulse }}\left\langle\hat{K}_{\mathbf{k}}^{+}+\hat{K}_{\mathbf{k}}^{-}\right\rangle \sin \left(2 \omega_{\mathbf{k}} t+\varphi_{\mathbf{k}}\right) \\
& +2 i V_{\mathbf{k}} \tau_{\text {pulse }}\left\langle\hat{K}_{\mathbf{k}}^{+}-\hat{K}_{\mathbf{k}}^{-}\right\rangle \cos \left(2 \omega_{\mathbf{k}} t+\varphi_{\mathbf{k}}\right),
\end{aligned}
$$

where the light-induced phase is

$$
\varphi_{\mathbf{k}}=\delta \omega_{\mathrm{R}} \tau_{\text {pulse }} \frac{1-\xi_{\mathbf{k}} \gamma_{\mathbf{k}}}{\sqrt{1-\gamma_{\mathbf{k}}^{2}}}
$$

To arrive at this results we used that the light-induced modification of the exchange interaction is small compared to the exchange constant itself, i.e., $\Delta J \ll J \Rightarrow \delta \omega_{\mathrm{R}} \ll \Omega$ and $\delta \omega_{\mathrm{R}} \tau_{\text {pulse }} \ll 1$ and kept only the first nontrivial terms linear in $\delta \omega_{\mathrm{R}}$. In equilibrium $V_{\mathbf{k}}=0$ and hence it follows that $K_{\mathbf{k}}^{z}(t)=$ $\left\langle\hat{K}_{\mathbf{k}}^{z}\right\rangle, \hat{K}_{\mathbf{k}}^{ \pm}(t)=0$. Substituting Eqs. (29) and (30) into Eq. (24) and using Eq. (23) we can formulate the following expression for the time dependence of the longitudinal component of the Néel vector:

$$
\left\langle L_{z}(t)\right\rangle=N S-\Delta L(0)-\delta L(t)
$$

where

$$
\Delta L(0)=\sum_{\mathbf{k}}\left(\frac{2\left\langle\hat{K}_{\mathbf{k}}^{z}\right\rangle}{\sqrt{1-\gamma_{\mathbf{k}}^{2}}}-1\right)
$$

and

$$
\delta L(t)=4 \tau_{\text {pulse }} \sum_{\mathbf{k}} \frac{\gamma_{\mathbf{k}} V_{\mathbf{k}}}{\sqrt{1-\gamma_{\mathbf{k}}^{2}}}\left\langle\hat{K}_{\mathbf{k}}^{z}\right\rangle \sin \left(2 \omega_{\mathbf{k}} t-\varphi_{\mathbf{k}}\right) .
$$

A number of remarks are in place here. First, we observe that the static value of the Néel vector is reduced as compared to the case of maximally aligned spins in each sublattice. This is directly related to the fact that the ground state is dressed by magnons. As is well-known for the quantum antiferromagnet, this is even true in the ground state at $T=0$, when no thermally induced magnons are present and $\left\langle\hat{K}_{\mathbf{k}}^{z}\right\rangle=1 / 2$, which is a consequence of the fact that $\hat{L}_{z}$ and $\hat{H}_{0}$ do not commute. Second, $\delta L(t)$ depends on $K_{\mathbf{k}}^{+}(t)+K_{\mathbf{k}}^{-}(t)$ [Eq. (29)] and does not require explicit knowledge of the initial state. In particular, it is sufficient to know only the equilibrium value of $\left\langle\hat{K}_{\mathbf{k}}^{z}\right\rangle$. Hence, our results are valid also at elevated temperature as long as the harmonic approximation is sufficiently accurate at the temperature and wavelength considered. It is also instructive to note that the time dependence of the correlators in Eq. (24), with account of the formulas in Eq. (29) takes the following form:

$$
\sum_{j, \delta}\left\langle\hat{S}_{j}^{z} \hat{S}_{j+\delta}^{z}\right\rangle \approx-\frac{1}{2} z_{N} N S^{2}+z_{N} S \Delta L(0)+z_{N} S \delta L(t) .
$$

\section{G. Effective macroscopic theory of $2 \mathrm{M}$ dynamics}

In the previous section, we have introduced magnon-pair operators to facilitate a microscopic description of $2 \mathrm{M} d y-$ namics in the harmonic approximation. In this section, we will focus on deriving an effective macroscopic theory of $2 \mathrm{M}$ dynamics, which supplements the standard Landau-Lifshitz equations on femtosecond timescales. To this end, we employ again the magnon-pair operators. In particular, analogous to what has been widely used for the dynamics of quantum spins, we utilize coherent states for magnon pair operators to derive effective macroscopic equations of motion for the dynamics of the antiferromagnetic vector.

To introduce the effective macroscopic variables we note that, according to Eq. (28) the wavefunction after the photoexcitation can be expressed as

$$
\left|\Psi_{\mathrm{AF}}(t)\right\rangle=\prod_{\mathbf{k}} \hat{U}_{\mathbf{k}}(t, 0)|\Psi(0)\rangle,
$$

where the wave function $|\Psi(0)\rangle$ describes the initial state. If the system was initially in the ground state $\left|\Psi_{\mathrm{AF}}(0)\right\rangle=$ $\prod_{\mathbf{k}}\left|0_{\uparrow \mathbf{k}}\right\rangle\left|0_{\downarrow-\mathbf{k}}\right\rangle$, then, after the pump pulse the wave function in Eq. (36) can be represented as the direct product $|\Psi(t)\rangle=\prod_{\mathbf{k}}\left|\mu_{\mathbf{k}}\right\rangle$ of the so-called Perelomov's coherent 
states:

$$
\left|\mu_{\mathbf{k}}\right\rangle=\sqrt{1-\left|\mu_{\mathbf{k}}\right|^{2}} \sum_{n=0}^{\infty} \mu_{\mathbf{k}}^{n}\left|n_{\mathbf{k}}\right\rangle\left|n_{\mathbf{k}}\right\rangle,
$$

where the parameter $\mu_{\mathbf{k}}$ is

$$
\mu_{\mathbf{k}}=i \tanh \left(\delta \omega_{\mathrm{R}} \tau_{\text {pulse }} \frac{\xi_{\mathbf{k}}-\gamma_{\mathbf{k}}}{\sqrt{1-\gamma_{\mathbf{k}}^{2}}}\right) e^{-2 i \omega_{\mathbf{k}} t+i \varphi_{\mathbf{k}}} .
$$

The values of the observables in state $\left|\Psi_{\mathrm{AF}}(t)\right\rangle$ are then obtained by using the general expressions for the averaged values of $\hat{K}$ operators [38]

$$
\begin{aligned}
\left\langle\mu_{\mathbf{k}}\left|\hat{K}_{\mathbf{k}}^{-}\right| \mu_{\mathbf{k}}\right\rangle & =\frac{\mu_{\mathbf{k}}}{1-\left|\mu_{\mathbf{k}}\right|^{2}}, \quad\left\langle\mu_{\mathbf{k}}\left|\hat{K}_{\mathbf{k}}^{+}\right| \mu_{\mathbf{k}}\right\rangle=\frac{\mu_{\mathbf{k}}^{*}}{1-\left|\mu_{\mathbf{k}}\right|^{2}}, \\
\left\langle\mu_{\mathbf{k}}\left|\hat{K}_{\mathbf{k}}^{z}\right| \mu_{\mathbf{k}}\right\rangle & =\frac{1}{2} \frac{1+\left|\mu_{\mathbf{k}}\right|^{2}}{1-\left|\mu_{\mathbf{k}}\right|^{2}} .
\end{aligned}
$$

The coherent states in Eq. (37), like the coherent states in optics, are the closest quantum states to a classical description of the magnonic field. The set of the coherent parameters $\mu_{\mathbf{k}}$ can thus be considered as proper variables representing the ultrafast spin dynamics of antiferromagnets at a macroscopic level. The equations of motion for these parameters are obtained in a quasiclassical limit as [39]

$$
\partial_{t} \mu_{\mathbf{k}}=\left\{\mu_{\mathbf{k}}, H_{\text {class }}\left(\mu_{\mathbf{k}}, \mu_{\mathbf{k}}^{*}\right)\right\},
$$

where the Poisson brackets $\{\ldots\}$ means

$$
\{A, B\}=\frac{\left(1-\left|\mu_{\mathbf{k}}\right|^{2}\right)^{2}}{i \hbar}\left(\frac{\partial A}{\partial \mu_{\mathbf{k}}} \frac{\partial B}{\partial \mu_{\mathbf{k}}^{*}}-\frac{\partial A}{\partial \mu_{\mathbf{k}}^{*}} \frac{\partial B}{\partial \mu_{\mathbf{k}}}\right) .
$$

The classical Hamiltonian, $H_{\text {class }}\left(\mu_{\mathbf{k}}, \mu_{\mathbf{k}}^{*}\right) \equiv \prod_{\mathbf{k}}\left\langle\mu_{\mathbf{k}}\right|\left(\hat{H}_{0}+\right.$ $\delta \hat{H}) \prod_{\mathbf{k}}\left|\mu_{\mathbf{k}}\right\rangle$, is calculated by substituting the expressions (39) into (21). We obtain

$$
\begin{aligned}
H_{\text {class }}\left(\mu, \mu^{*}\right)= & \langle\mu|\hat{H}| \mu\rangle=\hbar \sum_{\mathbf{k}}\left(\omega_{\mathbf{k}}+\delta \omega_{\mathbf{k}} f(t)\right) \frac{1+\left|\mu_{\mathbf{k}}\right|^{2}}{1-\left|\mu_{\mathbf{k}}\right|^{2}} \\
& +V_{\mathbf{k}} f(t) \frac{\mu_{\mathbf{k}}+\mu_{\mathbf{k}}^{*}}{1-\left|\mu_{\mathbf{k}}\right|^{2}} .
\end{aligned}
$$

Recalling the Hamiltonian in Eq. (42), the quasiclassical equations of motion for the parameters $\mu_{\mathbf{k}}$ take the following form:

$$
i \hbar \partial_{t} \mu_{\mathbf{k}}=2 \hbar\left(\omega_{\mathbf{k}}+\delta \omega_{\mathbf{k}} f(t)\right) \mu_{\mathbf{k}}+\hbar V_{\mathbf{k}} f(t)\left(1+\mu_{\mathbf{k}}^{2}\right) .
$$

Using (39), we can directly relate the longitudinal dynamics of the Néel vector with the coherent parameters. Combining Eqs. (39), (32), and (23), we obtain

$$
L_{z}=L_{z}(0)-\sum_{\mathbf{k}} \frac{\gamma_{\mathbf{k}}}{\sqrt{1-\gamma_{\mathbf{k}}^{2}}} \frac{2 \operatorname{Re} \mu_{\mathbf{k}}}{1-\left|\mu_{\mathbf{k}}\right|^{2}} .
$$

Purely longitudinal dynamics of the Néel vector, i.e., dynamics that does not induce any change of the total magnetization cannot be described by the standard Landau-Lifshitz equations. Therefore, additional macroscopic variables are required. To this end, we propose to use the parameters $\mu_{\mathbf{k}}$ to characterize short-range spin correlations and femtosecond scale dynamics.
Equations (43) together with the relations (44) and the standard Landau-Lifshitz equations for magnetic sublattices form a closed set of dynamic equations for macroscopic variables. Note that, however, quantum and classical spin dynamics can be disentangled, since they occur at different timescales. For example, in $\mathrm{KNiF}_{3}$, the longitudinal oscillations of $L_{z}$ take place on a subpicosecond timescale when the orientation of the Néel vector can be considered static. On the other hand, in the same material the classical precessional spin dynamics can be observed on a characteristic time-scale of 100 ps [14].

Furthermore, using the quasiclassical equation of motion, Eq. (43) and the Poisson brackets Eq. (41) we can define a generalized force as the conjugate variable of the coherent state variable $\mu_{\mathbf{k}}$ :

$$
H_{\mu_{\mathbf{k}}}=\left(1-\left|\mu_{\mathbf{k}}\right|^{2}\right)^{2} \frac{\partial H_{\text {class }}}{\partial \mu_{\mathbf{k}}^{*}} .
$$

The prefactor $\left(1-\left|\mu_{\mathbf{k}}\right|^{2}\right)^{2}$ arises because of the curvature of the hyper-unit sphere. The generalized force defined here is the mathematical analog of the effective magnetic field in the Landau-Lifshitz equations. It allows us to write Eq. (43) compactly as

$$
i \hbar \partial_{t} \mu_{\mathbf{k}}=H_{\mu_{\mathbf{k}}} .
$$

This representation is very useful since it enables the treatment of $2 \mathrm{M}$ dynamics on a purely phenomenological basis, without resorting to a specific microscopic model, as we will exploit in Sec. II H.

The parameter $\mu_{\mathbf{k}}$ in Eq. (38) is similar to the parameter of the coherent state (states corresponding to minimal uncertainity) often used in quantum optics. Its modulus is related to the average number of magnon pairs:

$$
\overline{n_{\mathbf{k}}}=\frac{\left|\mu_{\mathbf{k}}\right|^{2}}{1-\left|\mu_{\mathbf{k}}\right|^{2}},
$$

and to the probability to observe $n_{\mathbf{k}}$ magnon state in each of the correlated magnon modes:

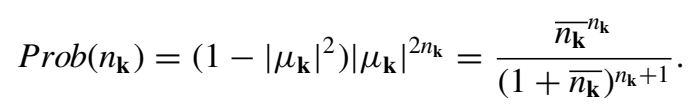

The values of $\mu_{\mathbf{k}}$ are related directly with the amplitude and phase of the oscillation of the Néel vector, as it can be seen from the expression (44).

To conclude this section, we mention that the quasiclassical Eq. (43), or equivalently Eq. (46), allows to take into account the main features of the subpicosecond description of the antiferromagnetic dynamics. For example, it can reproduce the interference between magnonic oscillations induced by two delayed pump pulses, experimentally observed in Ref. [23]. To illustrate this aspect, we consider the quantum dynamics induced by two subsequent pulses delayed in time by $t_{\text {delay }}$, so that $f(t)=\delta(t)+\delta\left(t-t_{\text {delay }}\right)$. In the initial state, $\mu_{\mathbf{k}}=0$. As it follows from Eq. (43), after the second pulse

$$
\mu_{\mathbf{k}}(t)=2 i \tanh V_{\mathbf{k}} \cos ^{2}\left(\omega_{\mathbf{k}} t_{\text {delay }}\right) e^{-2 i \omega_{\mathbf{k}} t}, \quad t \geqslant t_{\text {delay }},
$$

and, correspondingly, the oscillation amplitude in Eq. (66) acquires an additional factor which depends on the time delay in the following way:

$$
\delta L \Rightarrow 2 \delta L \cos ^{2}\left(\Omega t_{\text {delay }}\right) .
$$


Equation (50) shows that depending on $t_{\text {delay }}$ the amplitude of the quantum oscillations may be doubled (when $t_{\text {delay }}=$ $\pi / \Omega$ ) or vanish (when $t_{\text {delay }}=\pi / 2 \Omega$ ) as a result of constructive/destructive interference.

\section{H. Pump polarization dependence}

Earlier experiments demonstrated that the initial phase of the oscillations of the antiferromagnetic vector triggered by photoexcitation of the $2 \mathrm{M}$ mode depends on the polarization of the pump [23]. In this section, we try to reveal the origin of such a dependence, based on our microscopic formalism and on phenomenology.

It follows from our microscopic model [see Eq. (4) and the discussion in Sec. II D] that for a given orientation of the electric field of light $\mathbf{E}$, the initial phase of the oscillations of $\mathbf{L}$ is directly related to the sign of $\Delta J$ : an enhancement and a reduction of $J$ generate oscillations of the order parameter with opposite sign. In fact, the term containing $V_{\mathbf{k}}$ in Eqs. (8) and (22), which is responsible for the excitation of magnon pairs, depends linearly on $\Delta J$. Moreover Eq. (34) shows that $\delta L(t) \propto V_{\mathbf{k}}$ and hence a change in the sign of $\Delta J$ induces a modification of the sign of the amplitude of the antiferromagnetic vector. In our experiment, the sign of $\Delta J$ is positive and constant, but it could be negative if a pump photon energy bigger than the band-gap was employed [i.e., $\hbar \omega>U$, see Eq. (4)]. In addition, Eq. (34) contains a phase factor $\varphi_{k}$ defined in terms $\delta \omega_{R}$ [see Eq. (31)], which depends on $\Delta J$ as well. For a simple cubic lattice, Eq. (4) shows that the sign of $\Delta J$ is unaffected by rotating the polarization between directions parallel to different crystallographic axes. Thus within this approximation the phase of the signal is independent of the light polarization. However, a magnetoelastic strain $[40,41]$ or the asymmetry of the electronic orbitals due to spin-orbit interactions can further split degeneracy of $\Delta J$ depending on the orientation of the electric field of the pump with respect to the equilibrium orientation of spins. As a result, the effect of light on spins should be different in the cases when the pump polarization is parallel or perpendicular to the quantization axis (equilibrium orientation of spins). In such a situation, symmetry allows the phase to be different for different orientations of the electric field, because the modification of the exchange interaction induced by light with the electric field parallel to the equilibrium orientation of spins $\Delta J_{\|}$differs from the effect obtained if the polarization of light is rotated by $90^{\circ}$ with respect to the spin direction, i.e., $\Delta J_{\|} \neq \Delta J_{\perp}$.

Our description of the light-induced spin dynamics has so far neglected any anisotropy (i.e., $\Delta J_{\|}=\Delta J_{\perp}$ ). Considering now this anisotropic contribution, the amplitude and phase of the oscillations can be written as

$$
\begin{aligned}
\Delta L & =\frac{2}{3 \pi^{3}}\left(\delta \omega_{\mathrm{R}}^{\perp} e_{\perp}^{2}+\delta \omega_{\mathrm{R}}^{\|} e_{\|}^{2}\right)(\Delta k a)^{3} \tau_{\text {pulse }}, \\
\varphi & \approx\left(\delta \omega_{\mathrm{R}}^{\perp} e_{\perp}^{2}+\delta \omega_{\mathrm{R}}^{\|} e_{\|}^{2}\right) \tau_{\text {pulse }},
\end{aligned}
$$

where $\delta \omega_{\mathrm{R}}^{\perp}$ and $\delta \omega_{\mathrm{R}}^{\|}$represent the components of the modification of the light-matter interaction along a direction perpendicular and parallel to the equilibrium orientation of spins, respectively. Here the components $e_{\perp}$ and $e_{\|}$of the unit vector of light polarization are projected to the directions perpendicular and parallel to the Néel vector.

It is clear from Eq. (51) that controlling the light polarization allows one to manipulate the phase and amplitude of the oscillations. The variation of the phase is much more pronounced than the modification of the amplitude. The maximal variation of phase and amplitude $\propto\left(\delta \omega_{R}^{\|}-\delta \omega_{R}^{\perp}\right)$ can be achieved by rotating the light polarization from the parallel $\left(e_{\|}=1, e_{\perp}=0\right)$ to the perpendicular $\left(e_{\|}=0, e_{\perp}=1\right)$ configuration. Any rotation of the light polarization which preserves the relation between $e_{\|}^{2}$ and $e_{\perp}^{2}$ (e.g., within the plane perpendicular to the Néel vector) has no effect on the phase of the Néel vector oscillations.

A more general way to describe the effects of the polarization dependence of the longitudinal oscillations of $\mathbf{L}$ is based on a phenomenological modeling of the light-matter interaction. This approach does not specify microscopic mechanisms and takes into account just the symmetry of the sample $[24,25]$. The main idea consists in showing that the observed polarization dependence of the photoinduced change of the correlation function $\left\langle S^{\Uparrow} S^{\Downarrow}\right\rangle$ is allowed by the symmetry of the crystal. In particular, the $2 \mathrm{M}$ process in antiferromagnets can be described by means of the following phenomenological potential:

$$
\Phi=\chi_{j k l m} E_{j} E_{k}\left\langle S_{l}^{\Uparrow} S_{m}^{\Downarrow}\right\rangle
$$

where $\chi_{j k l m}$ represents a fourth rank magneto-optical polar tensor and plays the role of magneto-optical susceptibility. $E_{j, k}$ are the amplitude components of the electric field of the pump beam and $\left\langle S_{l}^{\Uparrow} S_{m}^{\Downarrow}\right\rangle$ is the correlation function between spins belonging to different sublattices.

The tensor $\hat{\chi}$ reflects the symmetry of light-matter interaction and is obviously invariant under permutation of the first two indices, $\chi_{j k l m}=\chi_{k j l m}$. The permutation of the second pair of indices is related to the permutation of the magnetic sublattices and thus should be treated according to the magnetic symmetry of the system. Note that Eq. (52) is also a phenomenological description valid for photoinduced magnetic order in paramagnetic media. To understand how light acts on a magnetically ordered medium, it is important to observe that the structure of the $\chi_{j k l m}$ tensor is governed by the symmetry of the magnetically ordered phase, which is lower than the symmetry of the paramagnetic phase. For the case, of $\mathrm{KNiF}_{3}$ studied in Ref. [23], the crystallographic point group is $m 3 m$, however the magnetic order lowers the symmetry of the medium down to $4 / \mathrm{mmm}$, where the fourfold axis is along the antiferromagnetic vector. Alternatively, the effect of light on the spin-correlation function in a magnetically ordered medium can be described as a higher-order effect:

$$
\Phi=\chi_{j k l m n o} E_{j} E_{k}\left\langle S_{l}^{\Uparrow} S_{m}^{\Downarrow}\right\rangle L_{n} L_{o},
$$

where $\chi_{j k l m n o}$ is a sixth rank tensor for the $m 3 m$ crystallographic point group and the form of tensor $\chi_{j k l m}$ for the $4 / \mathrm{mmm}$ point group can be found as $\chi_{j k l m}=\chi_{j k l m n o} L_{n} L_{o}$.

Analyzing the relations between the tensor components of the $4 / \mathrm{mmm}$ point group, [42] we note the following 
nonvanishing components of $\hat{\chi}$ in Voigt notations: ${ }^{2} \chi_{11}=$ $\chi_{22}=\chi_{12}=\chi_{21}, \chi_{33}, \chi_{13}=\chi_{23}, \chi_{31}=\chi_{32}, \chi_{44}=\chi_{55}$, and $\chi_{66}$. This means that the function in Eq. (52) can be written as

$$
\begin{aligned}
\Phi= & \chi_{11}\left(E_{x}^{2}+E_{y}^{2}\right)\left(\left\langle S_{x}^{\Uparrow} S_{x}^{\Downarrow}\right\rangle+\left\langle S_{y}^{\Uparrow} S_{y}^{\Downarrow}\right\rangle\right)+\chi_{33} E_{z}^{2}\left\langle S_{z}^{\Uparrow} S_{z}^{\Downarrow}\right\rangle \\
& +\chi_{13}\left(E_{x}^{2}+E_{y}^{2}\right)\left\langle S_{z}^{\Uparrow} S_{z}^{\Downarrow}\right\rangle+\chi_{31} E_{z}^{2}\left(\left\langle S_{x}^{\Uparrow} S_{x}^{\Downarrow}\right\rangle+\left\langle S_{y}^{\Uparrow} S_{y}^{\Downarrow}\right\rangle\right) \\
& +\chi_{66} E_{x} E_{y}\left\langle S_{x}^{\Uparrow} S_{y}^{\Downarrow}\right\rangle+\chi_{44} E_{z}\left(E_{x}\left\langle S_{x}^{\Uparrow} S_{z}^{\Downarrow}\right\rangle+E_{y}\left\langle S_{y}^{\Uparrow} S_{z}^{\Downarrow}\right\rangle\right)
\end{aligned}
$$

where we assumed the additional symmetry of the correlations $\left\langle S_{l}^{\Uparrow} S_{m}^{\Downarrow}\right\rangle=\left\langle S_{m}^{\Uparrow} S_{l}^{\Downarrow}\right\rangle$. The analysis of the function in Eq. (52) for a given polarization state can reveal which correlations could be excited. For this purpose, we need to express $\left\langle S_{l}^{\Uparrow} S_{m}^{\Downarrow}\right\rangle$ in terms of parameters of coherent states $\mu_{\mathbf{k}}$. As the density of light-induced magnonic states is peaked near $\mathbf{k} \approx \mathbf{k}_{\mathrm{R}}$, we can limit our description to $\mu_{\mathbf{k}_{\mathrm{R}}} \equiv \mu_{R}$. Moreover, as the pump pulse does not generate one-magnon excitations, all nondiagonal correlations $\left\langle S_{x}^{\Uparrow} S_{y}^{\Downarrow}\right\rangle,\left\langle S_{x}^{\Uparrow} S_{z}^{\Downarrow}\right\rangle$ etc vanish. Taking into account that the light-induced contribution to the correlation functions can be written as (introducing the phenomenological constant $A$ )

$$
\begin{gathered}
\left\langle S_{z}^{\Uparrow} S_{z}^{\Downarrow}\right\rangle=A \frac{1+\left|\mu_{R}\right|^{2}}{1-\left|\mu_{R}\right|^{2}}+\frac{\mu_{R}+\mu_{R}^{*}}{1-\left|\mu_{R}\right|^{2}}, \\
\left\langle S_{x}^{\Uparrow} S_{x}^{\Downarrow}\right\rangle=\left\langle S_{y}^{\Uparrow} S_{y}^{\Downarrow}\right\rangle=\frac{1}{2}\left[(1-A) \frac{1+\left|\mu_{R}\right|^{2}}{1-\left|\mu_{R}\right|^{2}}-\frac{\mu_{R}+\mu_{R}^{*}}{1-\left|\mu_{R}\right|^{2}}\right],
\end{gathered}
$$

we get for the function in Eq. (54) the following phenomenological expression:

$$
\begin{aligned}
\Phi= & \frac{\mu_{R}+\mu_{R}^{*}}{1-\left|\mu_{R}\right|^{2}} E^{2}\left[\left(\chi_{13}-\chi_{11}\right) e_{\perp}^{2}+\left(\chi_{33}-\chi_{31}\right) e_{\|}^{2}\right] \\
& +\frac{1+\left|\mu_{R}\right|^{2}}{1-\left|\mu_{R}\right|^{2}} E^{2}\left\{\left[\chi_{13} A+\chi_{11}(1-A)\right] e_{\perp}^{2}\right. \\
& \left.+\left(\chi_{33} A+\chi_{31}(1-A)\right) e_{\|}^{2}\right\} .
\end{aligned}
$$

Using this phenomenological potential we can exploit Eq. (45) to evaluate the generalized force. At the leading order in the small parameter $\mu$, we obtain

$$
H_{\mu} \approx \frac{\partial \Phi}{\partial \mu_{R}} \approx E^{2}\left[\left(\chi_{13}-\chi_{11}\right) e_{\perp}^{2}+\left(\chi_{33}-\chi_{31}\right) e_{\|}^{2}\right] .
$$

For a short pulse, the value of the generalized force defines the initial amplitude and phase of $\Delta L$. From Eq. (60), it follows that the symmetry allows different values of $H_{\mu}$ for the parallel $\left(e_{\|}=1, e_{\perp}=0\right)$ and perpendicular $\left(e_{\|}=0, e_{\perp}=1\right)$ configurations. Considering Eq. (60), we can formulate the following predictions.

(1) If light propagates along the Néel vector, as in the case of $\mathrm{KNi}_{2} \mathrm{~F}_{4}, e_{\|}=0, \Delta L \propto H_{\mu} \propto\left(\chi_{13}-\chi_{11}\right)$ and does not depend upon the direction of light polarization.

(2) If light propagates perpendicularly to the Néel vector, as in the case of $\mathrm{KNiF}_{3}$, both $e_{\|}$and $e_{\perp}$ components of the polarization vector could be nonzero. The difference between two

\footnotetext{
${ }^{2}$ We use Voigt notation for pair indices: $x x \rightarrow 1, y y \rightarrow 2, z z \rightarrow 3$,
} $y z \rightarrow 4, z x \rightarrow 5, x y \rightarrow 6$. orthogonal polarization states $\mathbf{E} \|\left(e_{\|}, e_{\perp}\right)$ and $\mathbf{E} \|\left(-e_{\perp}, e_{\|}\right)$ is $\propto\left(\chi_{33}-\chi_{31}-\chi_{13}+\chi_{11}\right)\left(e_{\|}^{2}-e_{\perp}^{2}\right)$. It is maximal when light is polarized parallel/perpendicular to the Néel vector.

\section{Quantum aspects of $2 \mathrm{M}$ dynamics}

In the previous sections, we have introduced qualitative, microscopic and effectively macroscopic descriptions of $2 \mathrm{M}$ dynamics, respectively. In this section, we elaborate on these descriptions focusing on the quantum aspects of $2 \mathrm{M}$ dynamics and discuss the relation with squeezing of quantum noise discussed before in connection with $2 \mathrm{M}$ excitation. Finally, we elaborate on the role of quantum and thermal fluctuations.

A simple perspective on the quantum nature of $2 \mathrm{M}$ dynamics follows from analyzing the microscopic interactions involved. For homogenous spin precession in antiferromagnets, the classical calculation gives exactly the same resonance frequencies as the fully microscopic quantum derivation. In both cases the frequency is determined from the competition of anisotropy and inter-sublattice exchange interactions. For $2 \mathrm{M}$ dynamics, the situation is different. As discussed in Sec. II D and from the microscopic theory outlined above, the homogenous dynamics of the Néel vector arises from the time-dependent quantum oscillations between the states with different numbers of $2 \mathrm{M}$ excitations and, correspondingly, with different energies. Such oscillations are similar to Rabi oscillations.

Moreover, the quantum states (37) which govern dynamics of the Néel vector, have all the features of the entangled (nonlocal and nonseparable) quantum states. First, the state of the system is formed by the pairs of magnons belonging to different modes (spin up and spin down) and propagating in opposite directions, as can be seen from the following expression:

$$
\left|\mu_{\mathbf{k}}\right\rangle=\sqrt{1-\left|\mu_{\mathbf{k}}\right|^{2}} \sum_{n=0}^{\infty} \mu_{\mathbf{k}}^{n}\left|n_{\uparrow \mathbf{k}}\right\rangle\left|n_{\downarrow-\mathbf{k}}\right\rangle \delta\left(n_{\uparrow \mathbf{k}}-n_{\downarrow-\mathbf{k}}\right) .
$$

This means that, at least theoretically, the individual magnons from different modes can be detected separately. Thus, for each $\mathbf{k}$, the system can be considered as a bipartite. Second, Eq. (37) predicts correlated statistics of the $\left|n_{\uparrow \mathbf{k}}\right\rangle$ and $\left|n_{\downarrow-\mathbf{k}}\right\rangle$ states of the individual magnon modes with equal $n_{\uparrow \mathbf{k}}=$ $n_{\downarrow-\mathbf{k}}=n_{\uparrow \mathbf{k}}$ [see Eq. (48)]. In other words, although individual measurement of one magnon mode can detect the state with any possible $n_{\uparrow \mathbf{k}}$, the outcome of the combined (simultaneous) measurement of two magnon modes is limited to the states with the same $n_{\uparrow \mathbf{k}}$. This means that the state of the system cannot be represented as a product of independent pure states of each magnon modes (nonseparability).

Thus the observed oscillations between the vacuum states $\left|0_{\uparrow \mathbf{k}}\right\rangle\left|0_{\downarrow-\mathbf{k}}\right\rangle$ and excited states $\left|n_{\uparrow \mathbf{k}}\right\rangle\left|n_{\downarrow-\mathbf{k}}\right\rangle$ could be treated as indication of the entanglement between $\uparrow$ and $\downarrow$ magnon modes. These states are equivalent to the two-mode coherently correlated photon states [43]. In analogy with optics, where the entangled states are produced by parametric downconversion, correlations between two different magnons modes are established in the course of a second-order magnetic Raman process [17], which conserves the total spin of the system. So, oscillations of the Néel vector result from the 
quantum correlations between the spin states at the different magnetic sublattices and have no counterpart in the magnetic dynamics described by the Landau-Lifshitz equations. We note further that, in the particular case of femto-nanomagnons, i.e., magnons with $\mathbf{k} \approx \mathbf{k}_{\mathrm{R}}$, all the coherent states $\left|\mu_{\mathbf{k}}\right\rangle$ have almost the same frequency and phase with difference only in amplitude. This additional, "classical" coherence of the different coherent states results in an ensemble response obtained by the sum of contributions from different modes and it allows a macroscopic observation of the quantum effect via optical methods.

Next, we elaborate on another quantum aspect of twomagnon dynamics, which is the squeezing of quantum noise. The coherent state of the two-magnon mode has been identified as a squeezed state and the fluctuations of the total squared magnetization have been ascribed as the squeezing variable [24,25]. While we agree that the two-magnon dynamics can be interpreted as a squeezed state, we put forward a different variable as squeezing variable, as we will explain below. First, we note that there are different definitions of squeezed and coherent states [44], which are equivalent only for a harmonic oscillator described by single-mode bosonic operators. In particular, coherent single-mode photon states are simultaneously eigenstates of the annihilation operator and the minimum uncertainty states. In addition, the squeezing modifies the coherent states in a way which reduces quantum fluctuations of one of noncommuting observables below the uncertainty limit. However, in the case of Perelomov's states, such simple classification can be misleading. In particular, the coherent two-magnon states in Eq. (37) are neither eigenstates of the operator $\hat{K}^{-}$nor the minimum uncertainty states for arbitrary $\mu$ [45]. Second, usually squeezing is associated with transformation of one time-independent state into another time-independent state with reduced fluctuations, which does not apply to the description of time-resolved experiments where $\mu$ is time-dependent.

However, within a certain extent we can consider the coherent two-magnon states in Eq. (37) as squeezed, meaning that in these states the quantum fluctuations $\Delta S$ are reduced with respect to their value in the ground state. In particular, in the ground state, we have $\left\langle S_{A i}^{z}\right\rangle=-\left\langle S_{B j}^{z}\right\rangle=S-\Delta S$, where

$$
\Delta S \equiv \frac{\Delta L(0)}{N}=\frac{1}{N} \sum_{\mathbf{k}}\left(\frac{1}{\sqrt{1-\gamma_{k}^{2}}}-1\right) .
$$

As follows from our theory [see Eqs. (32) and (34)], the longitudinal oscillations of $L_{z}$ periodically reduce $\Delta S$ below the ground-state value. Thus $\Delta S$ is identified as the squeezing variable in this context. The definition of the local magnetization has instead some subtleties. In Refs. [24,25], the local magnetization operator is defined as $\mathbf{m}_{i}=g \mu_{\mathrm{B}}\left(\mathbf{S}_{i}^{\Uparrow}+\mathbf{S}_{i+\delta}^{\Downarrow}\right)$. Here, $\mathbf{S}_{i+\delta}^{\Downarrow}$ is the spin operator of a nearest neighbor relative to $\mathbf{S}_{i}^{\Uparrow}$, in the opposite sublattice. A particular choice for $\boldsymbol{\delta}$, e.g., $\boldsymbol{\delta}_{1}=a \mathbf{e}_{x}$ defines an observable with a lower symmetry than the Hamiltonian. The total magnetization $\mathbf{M}=\sum_{i} \mathbf{m}_{i}$ as well as the antiferromagnetic order parameter $\mathbf{L}$ and $\Delta S$ are independent of the choice of $\delta_{1}$, since these are global variables. However, different choices for $\boldsymbol{\delta}_{1}$ can give different results for the local fluctuations $\left\langle\mathbf{m}_{i}^{2}\right\rangle$. We argue that this merely reflects the choice of the observable rather than the intrinsic physics of the system. To illustrate this, by averaging over $\delta$, we obtain

$$
\begin{aligned}
\frac{1}{\left(g \mu_{\mathrm{B}}\right)^{2}} \sum_{i, \boldsymbol{\delta}}\left\langle\mathbf{m}_{i}^{2}\right\rangle & =\sum_{i, \boldsymbol{\delta}}\left\langle\left(\mathbf{S}_{i}+\mathbf{S}_{i+\delta}\right)^{2}\right\rangle \\
& =\sum_{i, \boldsymbol{\delta}}\left\langle\mathbf{S}_{i}^{2}\right\rangle+\left\langle\mathbf{S}_{i+\delta}^{2}\right\rangle+2\left\langle\mathbf{S}_{i} \cdot \mathbf{S}_{i+\delta}\right\rangle \\
& =N S(S+1)+2 \sum_{i, \boldsymbol{\delta}}\left\langle\mathbf{S}_{i} \cdot \mathbf{S}_{i+\delta}\right\rangle .
\end{aligned}
$$

The last term on the right-hand side is proportional to the total energy, which is conserved after the pulse and hence does not show the characteristic oscillation with the period of the $2 \mathrm{M}$ mode. Therefore $\left\langle\mathbf{m}_{i}^{2}\right\rangle$ for a given $\boldsymbol{\delta}_{1}$ may oscillate, but the terms with different $\delta_{1}$ cancel each other. Despite this subtlety due to the choice for $\delta_{1}$, we note that the claim of Ref. [25] is that $\left\langle\mathbf{m}_{i}^{2}\right\rangle$ is proportional to $\frac{1}{2} \sum_{j, \delta}\left\langle\hat{S}_{j}^{+} \hat{S}_{j+\delta}^{-}+\right.$ $\left.\hat{S}_{j+\delta}^{-} \hat{S}_{j}^{+}\right\rangle$. The time-dependent contribution of this term is proportional to $\hat{K}^{+}(t)+\hat{K}^{-}(t)$, in fact it has the opposite sign as compared to the longitudinal correlations due to total energy conservation. Hence, the same term is determining the quantum fluctuations in both interpretations. To further support the interpretation of $\Delta S$ as squeezing variable we recall the discussion in Ref. [35] on the analogy between quantum noise in antiferromagnets and the influence of zeropoint motion on the lattice degrees of freedom. For the latter, the quantum noise is characterized by the deviation from the classical position $\delta a=\sqrt{\left\langle\left(R_{j}-\left\langle R_{j}\right\rangle\right)^{2}\right\rangle}$, which is removed in the limit of infinite mass $(m \rightarrow \infty)$. Similarly, the quantum noise in antiferromagnets is characterized by fluctuations of spin $\Delta S$, and the total spin $S$ plays the role of a mass. So, when $S \rightarrow \infty, \Delta S \rightarrow 0$ and the spin system can be described with classical spins. To conclude the discussion of squeezing, we note that fundamental excitations can exhibit also a different kind of squeezing, completely of thermal origin. Experimental observations of thermal squeezing of lattice modes (but not of magnons) on the picosecond time-scale have been reported [46,47].

We conclude this section with a qualitative discussion on the role of quantum and thermal fluctuations. The main contribution to the quantum noise in the ground state originates from the long-wavelength magnons with $\mathbf{k} \rightarrow 0$ for which $\gamma_{\mathbf{k}} \rightarrow 1$. In addition, quantum fluctuations to the sublattice magnetization scales as $1 / 2 z_{N}$ [35], $\left(z_{N}\right.$ being the number of nearest neighbors) making it almost undetectable in three dimensional samples as $\Delta S$ is only a few percent of $S$. On the other hand, light-induced oscillations of the spin correlations [see Eq. (35)] are related to femto-nanomagnons with $\mathbf{k}$ close to the edges of the Brillouin zone. The number of magnons is proportional to the intensity of light and can thus be detected in macroscopic samples. In addition, while the observation of the quantum noise effects in the ground state demands special conditions (e.g., low temperature), the light-induced $2 \mathrm{M}$ oscillations [see Eq. (34)] can be induced even if the system was initially in a mixed state. We would like to underline that our theory requires the presence of local rather than long-range order. Our approach can thus be applied at finite temperature: 
since it is a model based on spin-wave theory it is applicable in the temperature regime $T \ll 4 T_{N}$ in $2 \mathrm{D}$ and was found to give rather accurate agreement even for temperatures above $T_{N}$ [19].

Although calculating the actual temperature dependence of the spin dynamics induced by the excitation of femtonanomagnons goes beyond the harmonic approximation considered here, we can gain some insight into the role of temperature by addressing the problem in terms of statistical ensemble. In particular, reminding that the dominant contribution to the $2 \mathrm{M}$ process originates from the regions close to the edges of the Brillouin zone, where the magnon density of states peaks, we can further simplify the formulas for the spin dynamics by restricting our interest to the small range $\Delta k \ll$ $\pi /(2 a)$ in the vicinity of the $R$ point. Here, $\gamma_{\mathbf{k}}, \xi_{\mathbf{k}} \propto(\Delta k a)$ and

$$
\begin{aligned}
& \omega_{\mathbf{k}} \approx \Omega\left[1-O\left(\Delta k^{2} a^{2}\right)\right], \quad \delta \omega_{\mathbf{k}} \approx \delta \omega_{\mathrm{R}}\left[1-O\left(\Delta k^{2} a^{2}\right)\right], \\
& V_{\mathbf{k}} \propto \delta \omega_{\mathrm{R}}(\Delta k a) .
\end{aligned}
$$

Hence, the relevant energy scale for the longitudinal dynamics is $\hbar \omega_{R} \sim 2 z J S$ defined by the high-wavevector magnons, leading to oscillations on the femtosecond timescale. This is consistent with both the time-domain observations reported here and previously [23-25,48,49] and with the extensive spontaneous Raman literature [17,21]. In fact all these experiments performed in a huge variety of compounds demonstrated that the energy of the two-magnon mode is determined by the exchange energy and by a minor correction due to magnon-magnon interactions. Since the phase $\varphi_{\mathbf{k}} \approx \varphi \equiv \delta \omega_{\mathrm{R}} \tau_{\text {pulse }}$ and the magnon frequency $\omega_{\mathbf{k}}$ show small dispersion, the contributions from all the modes to Eq. (35) are almost coherent. Summing the amplitudes we obtain the following expression for the longitudinal correlations

$$
\begin{aligned}
\sum_{j, \delta}\left\langle\hat{S}_{j}^{z} \hat{S}_{j+\delta}^{z}\right\rangle= & -\frac{1}{2} z_{N} N S^{2}+z_{N} S \Delta L(0) \\
& +z_{N} S 4 \pi\left(\frac{2 \Delta k a}{3 \pi}\right)^{3}\left\langle\hat{K}_{\mathrm{k}_{\mathrm{R}}}^{z}\right\rangle e^{-t / \tau_{\mathrm{d}}} \sin (2 \Omega t-\varphi),
\end{aligned}
$$

and for the time-dependent longitudinal Néel component

$$
\begin{aligned}
\delta L(t)= & \delta \omega_{\mathrm{R}} \tau_{\text {pulse }} 4 \pi\left(\frac{2 \Delta k a}{3 \pi}\right)^{3}\left\langle\hat{K}_{\mathrm{k}_{\mathrm{R}}}^{z}\right\rangle e^{-t / \tau_{\mathrm{d}}} \\
& \times \sin \left(2 \Omega t-\delta \omega_{\mathrm{R}} \tau_{\text {pulse }}\right) .
\end{aligned}
$$

where $\left\langle\hat{K}_{\mathrm{k}_{\mathrm{R}}}^{z}\right\rangle=1 / 2+n_{\mathrm{k}_{\mathrm{R}}}$, meaning that the expectation value of the $K$ operator is evaluated in the vicinity of the $R$ point. Here we introduce the decoherence time $\tau_{\mathrm{d}}(\Delta k)$ as a phenomenological parameter. ${ }^{3}$

\footnotetext{
${ }^{3}$ Note that this term has to be interpreted as an ensemble decoherence and not like a dephasing of the individual magnon modes involved in the $2 \mathrm{M}$ process [23]. The parameter $\tau_{\mathrm{d}}(\Delta k)$ represents the damping of the oscillations observable in a pump-probe experiment [23]. An accurate calculation of $\tau_{\mathrm{d}}(\Delta k)$ goes beyond the harmonic approximation and thus the scope of our paper.
}

Within the realm of the harmonic approximation, it holds that $n \ll 1$. Moreover, since $\hbar \omega_{R}>T_{N}$, the thermal excitation of the femto-nanomagnons is very small in any temperature regime investigated. Hence we can conclude that the main contribution to $\left\langle\hat{K}_{\mathrm{k}_{\mathrm{R}}}^{z}\right\rangle$ stems from the population of magnons due to quantum fluctuations, which are already present even in the ground state. It is important to underline that the experimental approach introduced below does not directly probe the ground state fluctuations themselves. In fact, the latter have a random phase relation, forbidding the realization of a macroscopic coherent ensemble response which is, on the other hand, triggered by the photoexcitaton. In the all-optical experiments presented here and in the literature [23,48,49], only the macroscopic ensemble dynamics with a uniquely defined phase can be detected.

\section{METHODS AND MATERIALS}

\section{A. Magneto-optical pump-probe setup}

For our experiments, we used a regeneratively amplified mode-locked Ti:sapphire system delivering $100 \mathrm{fs}$ pulses with central photon energy equal to $1.55 \mathrm{eV}$. The average power is $4 \mathrm{~W}$ and the repetition rate is $2 \mathrm{kHz}$. A $500-\mathrm{mW}$ fraction of the laser output is used to drive two noncollinear optical parametric amplifiers (NOPAs) operating in two different spectral ranges [50]. Both NOPAs are pumped by the second harmonic of the laser (i.e., $3.1 \mathrm{eV}$ ) and seeded by the whitelight continuum produced by focusing the $1.55-\mathrm{eV}$ beam into a sapphire plate. The amplified pulse from the first NOPA, which initiates the dynamics (pump), has a spectrum spanning the $2.45-1.75 \mathrm{eV}$ range and is compressed to nearly transform-limited duration (i.e., $8 \mathrm{fs}$ ) by a pair of custommade chirped mirrors. The amplified pulse, generated by the second NOPA (probe), covers the frequency range between 1.5 and $1.18 \mathrm{eV}$ and is compressed to nearly transform-limited duration (i.e., $13 \mathrm{fs}$ ) by a couple of fused silica prisms. The temporal resolution of the setup has been characterized by the cross-correlation frequency-resolved optical gating (XFROG) technique and was below 20 fs [51]. The pump and probe beams were focused on the sample by a spherical mirror down to approximately 100 - and $70-\mu \mathrm{m}$ spot sizes, respectively. It is important to note that while the pump beam impinged on the sample surface at normal incidence (see Fig. 4), the probe beam propagated at an angle $\left(<10^{\circ}\right)$ with respect to the pump and with electric field close to the in-plane axes of the samples. Consequently, in the case of $\mathrm{K}_{2} \mathrm{NiF}_{4}$ [see Fig. 4(b)] a nonvanishing component of the probe beam propagates at an angle with the $c$ axis. The measurements on $\mathrm{KNiF}_{3}$ were performed at a minimum temperature of $77 \mathrm{~K}$ in a liquid nitrogen cryostat. The high temporal resolution is preserved by using a very thin $(200 \mu \mathrm{m})$ fused silica window as optical access to the liquid-nitrogen-cooled-cryostat. The experiments on $\mathrm{K}_{2} \mathrm{NiF}_{4}$ required liquid helium cooling, given the lower Néel temperature $\left(\mathrm{T}_{N}=96 \mathrm{~K}\right.$ versus $\mathrm{T}_{N}=246 \mathrm{~K}$ in $\left.\mathrm{KNiF}_{3}\right)$. The optical windows of the liquid-helium-cooled cryostat were 1-mm-thick sapphire plates. We pre-compressed the laser pulses by changing the optical path through the fused silica prisms, in order to preserve the superior time-resolution of our apparatus. The temperature of the samples was monitored in both cases by a thermocouple placed on the sample holder. 


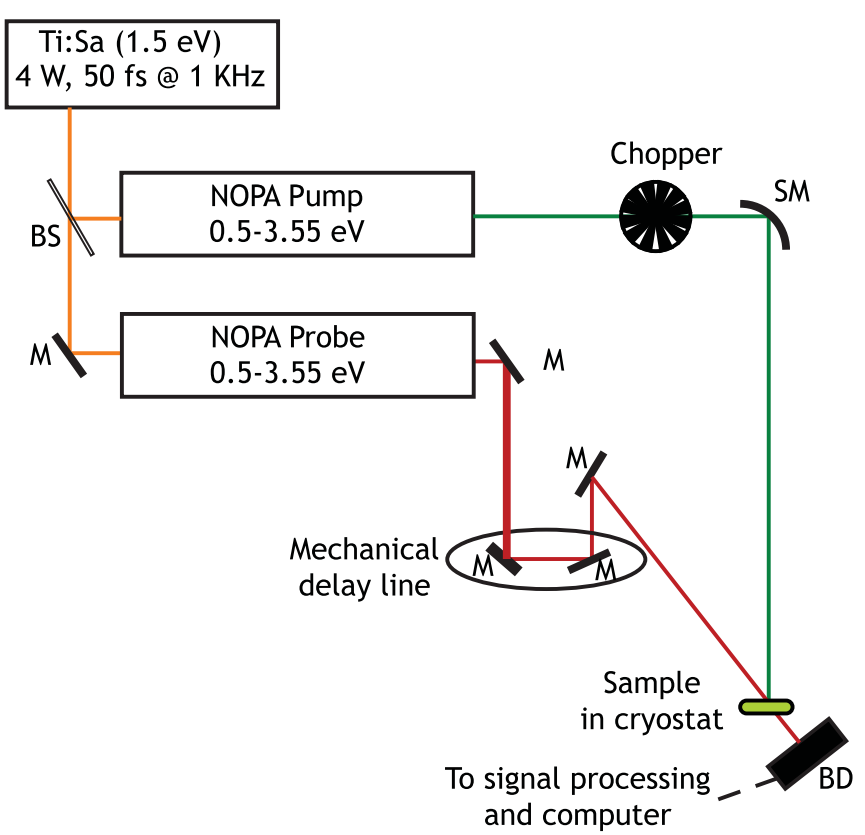

FIG. 5. Schematic representation of our setup. The main components are shown: mirrors (M), spherical mirrors (SM), beam splitters (BS) noncollinear optical parametric amplifiers (NOPA), and the balanced detector (BD). The polarization of the beams was linear and oriented along directions parallel to the crystal axes by means of half-wave plates and polarizers (not shown).

After interaction with the sample the linearly polarized probe beam was focused sent to a balanced detection setup to measure the polarization rotation. Note that in Ref. [14], the detection was based on the measurements of the ellipticity [52]. To achieve it, an additional quarter-wave plate had to be placed between the sample and the detector. In the present experiments the quarter-wave plate was removed from the scheme and the rotation of the polarization was detected. The linearly polarized transmitted probe is split by a Wollaston prism into two orthogonal linearly polarized beams and focused on a couple of balanced photodiodes. The Wollaston prism is rotated in order to equalize the probe intensities on the two photodiodes. The pump-induced imbalance of the signal registered by the two photodiodes is measured by a lock-in amplifier which is locked to the modulation frequency of the pump beam (i.e., $1 \mathrm{kHz}$ ). A schematic representation of the experimental setup is reported in Fig. 5. Our apparatus was able to detect rotations of the polarization down to $80 \mu \mathrm{deg}$. We did not employ a probe linearly polarized at $45^{\circ}$ with respect to the pump because the sample birefringence perturbs our sensitive polarization rotation scheme. Although the first detection of the dynamics of two-magnon mode in antiferromagnets $\mathrm{MnFe}_{2}$ and $\mathrm{FeF}_{2}$ was based on time-resolved measurements of differential transmissivity, a more recent work employed the method of time-resolved polarization rotation measurements [23]. Here we briefly review the physics of the probing mechanisms of the spins dynamics in antiferromagnets. In a linear light-matter interaction regime, the response of the media to the illumination is described in terms of dielectric permittivity tensor $\epsilon^{i j}$. If in an otherwise isotropic medium (i.e., $\epsilon^{x x}=\epsilon^{y y}=\epsilon^{z z}$ ) the spin correlation function $\left\langle\hat{S}_{i}^{\Uparrow} \hat{S}_{j}^{\Downarrow}\right\rangle$ experiences a modification, such variation can be detected by optical methods due to a contribution to the symmetric part of the dielectric permittivity [53] $\epsilon_{s}^{\lambda \nu}=\epsilon_{s}^{\nu \lambda}$ :

$$
\delta \epsilon_{s}^{\lambda \nu}=\sum_{i j} \sum_{\gamma \delta} \rho^{\lambda v \gamma \delta}\left\langle\hat{S}_{i}^{\gamma \Uparrow \hat{S}_{j}^{\delta \Downarrow}}\right\rangle,
$$

where $\rho^{\lambda v \gamma \delta}$ is a phenomenological polar forth-rank tensor, $i j$ describe lattice sites and $\lambda \nu \gamma \delta$ are spatial coordinate indices. This contribution affects the absorption and refraction coefficients of the material due to isotropic contribution to the dielectric permittivity (i.e., $\delta \epsilon^{x x}=\delta \epsilon^{y y}=\delta \epsilon^{z z}$ ), thus modifying the intensity, reflected, absorbed and transmitted light beams, as reported [24,25]. An emergence of anisotropic contributions $\left(\delta \epsilon^{x x} \neq \delta \epsilon^{y y} \neq \delta \epsilon^{z z}\right)$ would result in different absorption and refraction of a light beam linearly polarized along the $x, y$, and $z$ axes, respectively. Let's consider the propagation of a light beam along the $z$ axis. If $\Re \epsilon^{x x} \neq \Re \epsilon^{y y}$, the intensities of reflected beams polarized along the $x$ and $y$ axes, respectively, are different. On the other hand, in case $\Im \epsilon^{x x} \neq \Im \epsilon^{y y}$, the absorption experienced by beams polarized along the $x$ and $y$ axes, respectively, and consequently the transmitted intensities, differ. As reported in the literature [54], this inequality results in a polarization rotation of the probe beam which is proportional to the modification of order parameter or, as in our case, of the spin correlation function $\left\langle\hat{S}_{i}^{\Uparrow} \hat{S}_{j}^{\Downarrow}\right\rangle$.

In optically anisotropic media $\epsilon^{x x} \neq \epsilon^{y y} \neq \epsilon^{z z}$ the optical detection of the dynamics of the spin correlation function $\left\langle\hat{S}_{i}^{\Uparrow} \hat{S}_{j}^{\Downarrow}\right\rangle$ is more complex. In fact, even if the spin correlation function contributed isotropically to the dielectric permittivity $\delta \epsilon^{x x}=\delta \epsilon^{y y}=\delta \epsilon^{z z}$, it would follow that $\epsilon^{x x} / \epsilon^{y y} \neq\left(\epsilon^{x x}+\right.$ $\left.\delta \epsilon^{x x}\right) /\left(\epsilon^{y y}+\delta \epsilon^{y y}\right)$ and the dynamics of $\left\langle\hat{S}_{i}^{\Uparrow} \hat{S}_{j}^{\Downarrow}\right\rangle$ could still generate dynamics of the polarization rotation of the probe beam. Using the method of balanced detection, the intensity noise of laser sources can be greatly compensated and measurements of polarization rotation can be performed with an extreme sensitivity limited by the level of shot noise. In order to achieve the highest possible sensitivity, we employed the technique of polarization rotation [23]. We would like also to observe that since the response originates from the unbalance of $\epsilon^{x x}$ and $\epsilon^{y y}$ a probe beam polarized $45^{\circ}$ away from the crystal axes would maximize the signal. However, this configuration did not provide best the signal-to-noise ratio, because of a strong increase of the background noise, whose origin was not investigated in details. Therefore we empirically selected the probe polarization resulting in the best signal-to-noise ratio. The best direction was found to be approximately parallel to one of the crystallographic axes. More precisely, in the case of $\mathrm{KNiF}_{3}$ the polarization of the probe was approximately parallel to the $y(z)$ axis and in the case of $\mathrm{K}_{2} \mathrm{NiF}_{4}$ to the $x(y)$ axis. The degree of approximation is estimated to be of the order of $10^{\circ}$. A drawback of our approach concerns the interpretation of the experimental results. In time-resolved studies of the dynamics of the $\left\langle\hat{S}_{i}^{\Uparrow} \hat{S}_{j}^{\Downarrow}\right\rangle$ four different scenarios can originate an anisotropy leading to the polarization rotation: (a) the dynamics of the spin correlation induces an isotropic contribution to the dielectric permittivity $\left(\delta \epsilon^{x x}=\delta \epsilon^{y y}=\delta \epsilon^{z z}\right)$, but the medium is anisotropic in the 
unperturbed state $\left(\epsilon^{x x} \neq \epsilon^{y y} \neq \epsilon^{z z}\right)$. (b) The dynamics of the spin correlation induces an anisotropic contribution to the dielectric permittivity $\left(\delta \epsilon^{x x} \neq \delta \epsilon^{y y} \neq \delta \epsilon^{z z}\right)$, but the medium is isotropic in the unperturbed state $\left(\epsilon^{x x}=\epsilon^{y y}=\epsilon^{z z}\right)$. (c) The dynamics of the spin correlation induces an anisotropic contribution to the dielectric permittivity $\left(\delta \epsilon^{x x} \neq \delta \epsilon^{y y} \neq \delta \epsilon^{z z}\right)$, and the medium is anisotropic in the unperturbed state $\left(\epsilon^{x x} \neq\right.$ $\epsilon^{y y} \neq \epsilon^{z z}$ ). (d) Although the medium is isotropic in the unperturbed state $\left(\epsilon^{x x}=\epsilon^{y y}=\epsilon^{z z}\right)$ and the spin correlation induced an isotropic contribution as well $\left(\delta \epsilon^{x x}=\delta \epsilon^{y y}=\delta \epsilon^{z z}\right)$, the intense linearly polarized pump beam can induce an anisotropic transient linear birefringence of nonmagnetic origin.

In our experiment, the dynamics of the spin correlation is induced by the intense pump pulse: as reported in our earlier work [23], the photoinduced dynamics of $\left\langle\hat{S}_{i}^{\Uparrow} \hat{S}_{j}^{\Downarrow}\right\rangle$ is a linear function of the pump intensity and provides an anisotropic contribution to the dielectric permittivity $\left(\delta \epsilon^{x x} \neq\right.$ $\delta \epsilon^{y y} \neq \delta \epsilon^{z z}$ ). Therefore the measured signal in the cases scenario (a) and (b) and (c) is expected to be linear with respect to the pump intensity, while only (b) and (c) are relevant to our experiment. More specifically, (b) can be ruled out since both materials here investigated are anisotropic before the photoexcitation. The main difference between the two compounds concerns the origin of the anisotropy: it arises from the crystal structure in $\mathrm{K}_{2} \mathrm{NiF}_{4}$ (being thus insensitive to the Néel temperature), while it has magnetic origin in $\mathrm{KNiF}_{3}$ (sensitive to the Néel temperature). The last term, (d) can be neglected since it is expected to depend quadratically (or even with a higher degree of nonlinearity) on the intensity of the pump beam. This statement is motivated by the fact that photoinduced modification of the birefringence depends at the leading order linearly on the pump fluence, as the dynamics of the spin correlation function. Hence, the combined effect should display a nonlinear dependence on the excitation fluence, in contrast with the observation reported in Sec. VI.

The SR spectrum of $\mathrm{K}_{2} \mathrm{NiF}_{4}$ was measured in the backscattering geometry. The sample was excited by two different $\mathrm{CW}$ lasers, a diode with central photon energy of approximately $2.3 \mathrm{eV}$ and a He-Ne source $(\approx 1.9 \mathrm{eV})$. The power of the incident radiation on the sample was $220 \mu \mathrm{W}$ in the former case and $110 \mu \mathrm{W}$ in the latter. The backscattered light was collected by a $10 \mathrm{x}$ objective (numerical aperture $\approx 0.25$ ) and dispersed by a Horiba LabRam HR800 spectrometer. The detector was a cooled CCD camera, able to scan the Raman shift in the range from 200 to $700 \mathrm{~cm}^{-1}$. The sample was mounted on the cold finger of a liquid-nitrogen-cooled flow cryostat, held at a constant temperature of $70 \mathrm{~K}$. The Raman shift was calibrated and the intensities were normalized by employing the $520-\mathrm{cm}^{-1} \mathrm{Si}$ phonon peak measured under the same conditions.

\section{B. Materials}

We investigated two dielectric collinear antiferromagnets: the cubic $\mathrm{KNiF}_{3}$ and the layer-structured (i.e., $\left.2 \mathrm{D}\right) \mathrm{K}_{2} \mathrm{NiF}_{4}$. Our $\mathrm{KNiF}_{3}$ sample was a $340-\mu$ m-thick (100) single crystal, which has a perovskite crystal structure. Two equivalent $\mathrm{Ni}^{2+}$ sublattices are antiferromagnetically coupled below the Néel temperature $T_{\mathrm{N}}=246 \mathrm{~K}$ [14]. In the paramagnetic phase $\mathrm{KNiF}_{3}$ is described by the $m 3 m$ point group, while in the ordered phase it belongs to the $4 / \mathrm{mmm}$ group. The ultrafast dynamics of the short-wavelength magnons in this system has already been reported [23]. Here we discuss the dependence of the signal on the temperature and on the polarization of the pump beam, in comparison with the results obtained for the uniaxial antiferromagnet.

The structure of $\mathrm{K}_{2} \mathrm{NiF}_{4}$ consists of antiferromagnetic planes of $\mathrm{NiF}_{2}$ separated by $\mathrm{KF}$ planes, which is similar to the atomic arrangement of superconducting cuprates of $\mathrm{La}_{2} \mathrm{CuO}_{4}$ type [see Fig. 4(b)]. Our specimen is an $800-\mu$ m-thick single crystal cut perpendicular to the $c$ axis. This material orders at $\mathrm{T}_{N} \approx 96 \mathrm{~K}$. Also $\mathrm{K}_{2} \mathrm{NiF}_{4}$ belongs to the $4 / \mathrm{mmm}$ group in the antiferromagnetic phase, where the orientation of the fourfold axis is given by the orientation of antiferromagnetic vector. The dominant exchange interaction determines an antiparallel alignment of the $\mathrm{Ni}^{2+}$ spins in the $\mathrm{NiF}_{2}$ planes, via $180^{\circ} \mathrm{Ni}-\mathrm{F}-\mathrm{Ni}$ bonds $[30,55]$. Even neglecting the interplane exchange interaction between the $\mathrm{Ni}^{2+}$ ions in the ordered planes and the isolated $\mathrm{Ni}^{2+}$ ion between the planes (which is at least one order of magnitude weaker than the in-plane exchange coupling [30]), the bulk properties of this compound are properly described.

While for both these compounds the exchange interaction is taken into account by means of the nearest-neighbor Heisenberg interaction, the magnetocrystalline anisotropies strongly differ. In the case of $\mathrm{KNiF}_{3}$ a very weak cubic magnetic anisotropy with positive sign of the anisotropy constant determines the alignment of spins along the [001], [010], and [100] axes [27]. The size of the domains was reported to be on the mm-scale, so that the spot size of our focused laser beams $(70-100 \mu \mathrm{m})$ allows to interrogate a single domain in this material [56]. On the other hand the sublattice magnetization in $\mathrm{K}_{2} \mathrm{NiF}_{4}$ is parallel to the $c$ axis, due a single-axis anisotropy [30].

Raman spectroscopy investigations revealed the features of the $2 \mathrm{M}$ mode in $\mathrm{K}_{2} \mathrm{NiF}_{4}[18,19,57]$. At low temperature $(10 \mathrm{~K})$, the Raman shift is approximately $520 \mathrm{~cm}^{-1}$, corresponding to $v \approx 15.6 \mathrm{THz}$ (period $\approx 65 \mathrm{fs}$ ), while the linewidth (FWHM) is $100 \mathrm{~cm}^{-1}$, from which a lifetime on the order of $330 \mathrm{fs}$ is expected. The spectrum of this material shows also several Raman-active phonon modes [57], in particular a collective vibration with frequency in the $11-\mathrm{THz}$ range $\left(\approx 380 \mathrm{~cm}^{-1}\right)$. These observations have been confirmed by the measurement of the SR spectrum on our specimen of $\mathrm{K}_{2} \mathrm{NiF}_{4}$, reported in Fig. 6. Although the long-range magnetic properties are dramatically different for these compounds $[30,58]$, the experimental evidence concerning the magnons near the edges of the Brillouin zone in $\mathrm{K}_{2} \mathrm{NiF}_{4}$ are comparable to the case of $\mathrm{KNiF}_{3}$, as discussed in Sec. IV.

\section{TEMPERATURE DEPENDENCE OF THE FEMTO-NANOMAGNONS}

Differently from low-energy collective spin excitations with wave vector at the center of the Brillouin zone, the frequency of the $2 \mathrm{M}$ mode does not soften upon approaching the Néel point. Moreover, spontaneous Raman experiments have detected a peak at the characteristic frequency of the $2 \mathrm{M}$ excitation even when the temperature was higher than $T_{\mathrm{N}}$. This is common to basically all the antiferromagnets 


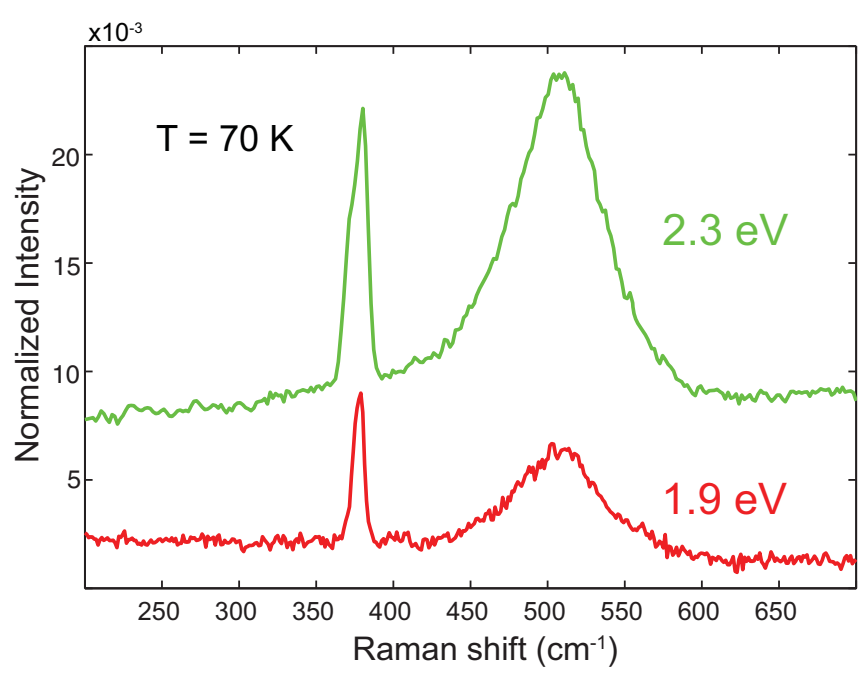

FIG. 6. The spontaneous Raman spectrum of $\mathrm{K}_{2} \mathrm{NiF}_{4}$, measured with 2.3- and 1.9-eV CW lasers. These photon energies are comparable with the ones employed in the pump-probe experiments. The power of the incident radiation on the sample was $220 \mu \mathrm{W}$ for the $2.3 \mathrm{eV}$ excitation, while it amounted to $110 \mu \mathrm{W}$ when the photon energy was $1.9 \mathrm{eV}$. The temperature was $70 \mathrm{~K}$.

investigated [21]. It was conventionally accepted that, the Raman signal is detected above the Néel temperature because short-range spin correlations

In contrast with SR experiments, the time-domain observations of the spin dynamics induced by optically generating the $2 \mathrm{M}$ mode have failed to reproduce this experimental trend in several materials $[23,25]$. To be more precise, although the temperature dependence of the frequency of the $2 \mathrm{M}$ mode did not reveal any noticeable softening [23], the amplitude of the femto-nanomagnonic oscillations decreased upon a temperature increase and no signal has ever been observed above the Néel temperature $[23,25]$. Therefore it has been suggested that long-range spin correlation play "an important, if not essential role" for the $2 \mathrm{M}$ process [25], pointing towards the possibility of a discrepancy in the results obtained employing the two different experimental approaches.

We study this open problem by measuring and comparing the temperature dependence of the spin dynamics observed in $\mathrm{KNiF}_{3}$ and $\mathrm{K}_{2} \mathrm{NiF}_{4}$. These materials have different magnetic anisotropies, therefore long-range spin correlations, which presumably affect the $2 \mathrm{M}$ process in these compounds, are also expected to be different. Note that the optical spectrum of the two compounds is almost identical, except for the fine-structure splitting of some $d$ - $d$ transitions, which is however too tiny to be resolved by broadband femtosecond laser pulses [55]. Consequently, a straightforward comparison of the results obtained with these two samples can be carried out only based on their magnetic properties.

Figure 7(a) reports the temperature dependence of the spin dynamics optically excited and detected in the time domain in $\mathrm{K}_{2} \mathrm{NiF}_{4}$. The corresponding measurements for $\mathrm{KNiF}_{3}$ are reported in Ref. [23]. Figure 7(b) reports the temperature dependence of the frequency of the $2 \mathrm{M}$ mode observed in the time domain in $\mathrm{KNiF}_{3}$ (blue dots) and $\mathrm{K}_{2} \mathrm{NiF}_{4}$ (green dots). In the latter case, it was possible to observe oscillations
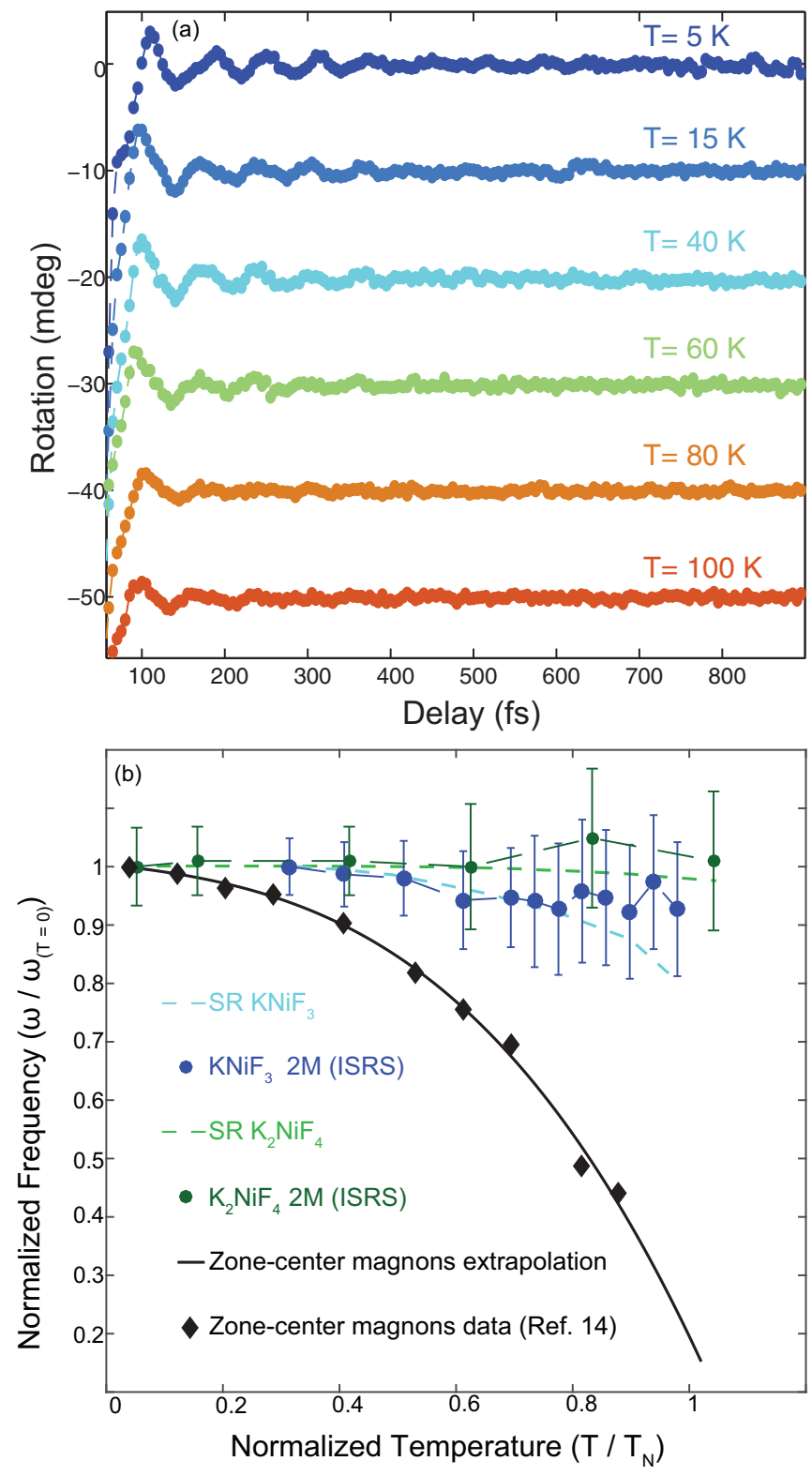

FIG. 7. Temperature dependence of normalized frequency of the two-magnon mode. (a) Spin dynamics as a function of the temperature measured in $\mathrm{K}_{2} \mathrm{NiF}_{4}$. The pump and probe photon energies were 1.9 and $1.3 \mathrm{eV}$, respectively. The fluence was set to $\approx 4.5 \mathrm{~mJ} / \mathrm{cm}^{2}$ and the pump beam was circularly polarized. (b) The frequency of the oscillations is estimated from the Fourier transform of the data. Following a procedure typically employed in the Raman literature, [57] the frequency of the oscillations is normalized to the value of the frequency detected at the lowest temperature. Note that the minimum temperature achieved for the two samples is different: $80 \mathrm{~K}$ for $\mathrm{KNiF}_{3}$ and $5 \mathrm{~K}$ in $\mathrm{K}_{2} \mathrm{NiF}_{4}$. The error bars are defined as half width half maximum of the two-magnon spectrum. The dashed lines represent the result of SR scattering experiments on $\mathrm{KNiF}_{3}$ [23] and $\mathrm{K}_{2} \mathrm{NiF}_{4}$ [57]. The black diamonds represent experimental results of the frequency of the low-energy magnons $\mathrm{KNiF}_{3}$ from Ref. [14]. The black continuous line was calculated using these results, to demonstrate the characteristic softening of the long-wavelength spin waves near the Néel point. 
also when the temperature was set slightly above the Néel point. Note that our results are consistent with experimental investigations of the temperature dependence of the $2 \mathrm{M}$ mode by means of SR spectroscopy [see dashed lines in Fig. 7(b)]. On the same panel, we plot the typical temperature dependence of the frequency of $\mathbf{k} \approx 0$ magnons in antiferromagnets [14], exhibiting the characteristic softening as the temperature of the sample approaches the Néel point. It is important to observe that also the long-wavelength magnons in $\mathrm{K}_{2} \mathrm{NiF}_{4}$ soften at $T_{\mathrm{N}}$, as demonstrated experimentally [59]. It is evident that the temperature dependence of the $2 \mathrm{M}$ frequency of both samples does not display any softening.

According to the well-established spin wave theory, the frequency of magnons near the center of the Brillouin zone is defined by both the effective magnetocrystalline anisotropy field and the exchange interaction [21]. On the other hand, the exchange energy only is relevant for the frequency of magnons near the edges of the Brillouin zone, which are the spin excitations involved in the $2 \mathrm{M}$ mode. The absence of any softening in the data set shown in Fig. 7(b) implies that the $2 \mathrm{M}$ excitation itself is not affected by the long-range order.

Thus the question naturally arises: why is the signal not observed well-above the Néel point in the time-domain as it occurs in the frequency-domain Raman spectroscopy? In our opinion, the explanation does not lie in the difference between generation of magnons via stimulated and spontaneous Raman scattering, but in the different detection of the $2 \mathrm{M}$ mode in frequency-resolved and time-resolved Raman experiments. In particular, even above the Néel point, where the net magnetic order parameter is vanishing, short-range spin-spin correlations are still present and a light beam can still scatter on $2 \mathrm{M}$ excitations. Relying on a spectral analysis of the scattered light beam, experimental signatures of the $2 \mathrm{M}$ excitations in the paramagnetic phase can be detected [21]. On the other hand, in the case of the time-resolved approach, the situation is different: the detection of the time dependence of the spin correlation is phase-sensitive. Once the long-range magnetic order is lost above the Néel temperature, the oscillations of the magnetic ensemble do not have the same phase, and thus average out. As a result, a time-resolved detection of coherent magnons at the edge of the Brillouin zone is not possible. We observe that even if a single pump-probe trace revealing the $2 \mathrm{M}$ mode was observed when the temperature was set slightly above the Néel point in $\mathrm{K}_{2} \mathrm{NiF}_{4}$ [see Fig. 7(a)], it does not mean that the whole sample volume probed in the measurement was in the paramagnetic phase. In fact, in the case of wide band-gap dielectric materials with poor thermal conductivity a mismatch between the temperature set value and the material temperature on the order of several Kelvin typically occurs.

Having established that only the short-range spin-spin correlations and zone-edge magnons are relevant to the femtonanomagnonics, we can also conclude that, in this particular dynamical regime, the magnetocrystalline anisotropy does not play an important role, since the properties of zoneedge magnons are dominated by the exchange interaction. However, the long-range magnetic properties are important for the magneto-optical detection of the zone-edge magnons in time-resolved experiment.

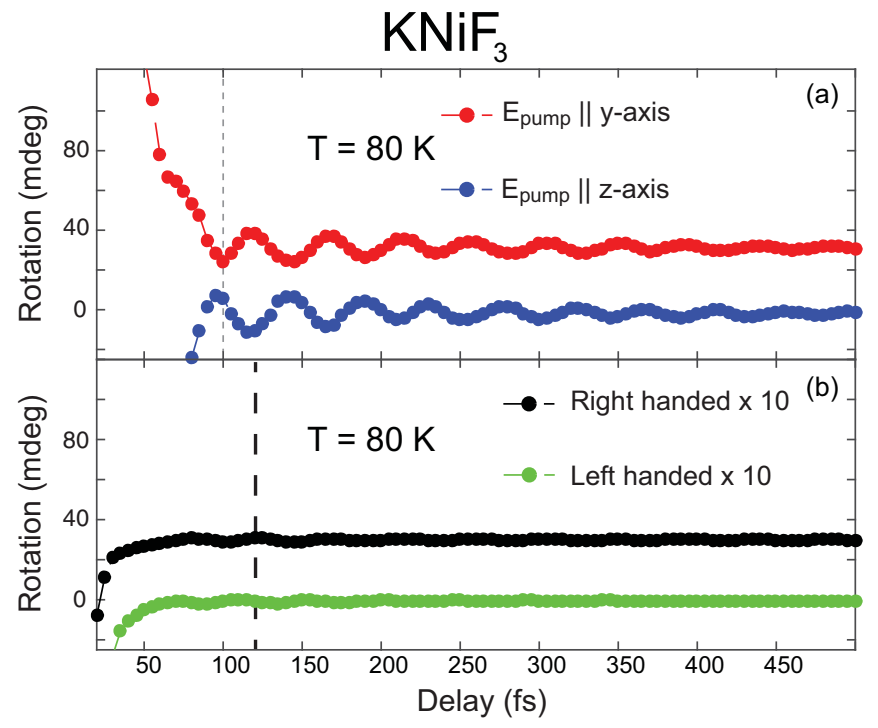

FIG. 8. ISRS measurements in $\mathrm{KNiF}_{3}$ using pump pulses with different linear (a) and circular (b) polarization. The measurements were performed at $80 \mathrm{~K}$. The pump and probe photon energies were 2.2 and $1.3 \mathrm{eV}$, respectively. The grey and black dashed lines highlight the phase relation among the different time traces in the case of linearly or circularly polarized pump beam, respectively. The experimental geometry is shown in Fig. 4(a). (a) The excitation beam was linearly polarized and the pump fluence was on the order of $10 \mathrm{~mJ} / \mathrm{cm}^{2}$. (b) The data (multiplied by a factor 10) here shown were obtained by employing circularly polarized pump beams. The fluence was on the order of $20 \mathrm{~mJ} / \mathrm{cm}^{2}$.

\section{PUMP POLARIZATION DEPENDENCE}

The effect of the pump-beam polarization on the amplitude and the phase of the oscillations of the antiferromagnetic vector $\mathbf{L}$ was reported for $\mathrm{KNiF}_{3}$ [23], but the origin has not been discussed in the literature yet. Here we explore the pump-polarization dependence of the femto-nanomagnonic signal in materials with very similar optical properties, but substantially different spin structures (i.e., $\mathrm{KNiF}_{3}$ and $\mathrm{K}_{2} \mathrm{NiF}_{4}$ ).

Let us first consider $\mathrm{KNiF}_{3}$. The ISRS dynamics reported in Fig. 8(a) shows that rotating the electric field of the linearly polarized pump beam from one axis to the other results in a $\pi$ shift of the phase of the oscillations. Since we employed laser beams linearly polarized along both the $y$ and $z$ axes, the configuration shown in Fig. 4(a) is a proper representation of the experiment: regardless of which domain is contributing to our signal ( $y$ or $z$ domain), we explored both the conditions of electric field parallel and perpendicular to spins. This is fully consistent with the well-known magnetic configuration of $\mathrm{KNiF}_{3}$, which consists of spins aligned along directions parallel to the crystallographic axes [14].

We analyze now the excitation induced employing circularly polarized light, for which the $z$ - and $y$-components of the electric field have a phase difference equal to $\pi / 2$. Note that for this polarization state the following property holds: $E_{y} E_{z}^{*}=-E_{z} E_{y}^{*}$. Making use of this properties it is possible to show that the light-matter interaction vanishes for both helicities (following an approach reported in the 
literature [23]) entailing that the excitation of the $2 \mathrm{M}$ mode is forbidden for circularly polarized laser pulses. This, in principle, is not surprising. In fact a circularly polarized optical beam accesses the antisymmetric components of the dielectric tensor, which are proportional to the odd powers in spin $[27,60]$. The $2 \mathrm{M}$ scattering is a process quadratically dependent on the spin and it is therefore described by the symmetric components of the dielectric tensor [17,21]. Therefore a purely circularly polarized beam cannot generate coherent femto-nanomagnons. However, a tiny although detectable magnonic oscillations were observed by pumping $\mathrm{KNiF}_{3}$ with a circularly polarized optical beam [see Fig. 8(b)]. This apparent discrepancy with the symmetry analysis is due to an imperfect polarization of the pump beam. The superior time resolution of our experiment is obtained by using broadband laser pulses $(F W H M \approx 40 \mathrm{~nm})$. The broadband quarter waveplate employed to generate the circular polarization state has a slightly different retardation for each spectral component of our pulses. Consequently, the polarization state of the beam after the waveplate is elliptical. Since an elliptically polarized beam can be described as the sum of a circularly and a linearly polarized beam, we ascribe the oscillations observed in Fig. 8(b) to the residual linear component. The suggested phenomenological model in Sec. II H predicts the polarization dependence of the laser-induced two-magnon oscillations in $\mathrm{KNiF}_{3}$; moreover we would also like to observe that, even in a fully isotropic medium, linearly polarized light $E_{j} E_{j}$ couples to specific components of spins $\left\langle S_{j} S_{j}\right\rangle$. A rotation of the polarization of the pump over 90 degrees would corresponds to the excitation of different spin components. This will result in a sign change of the polarization rotation induced by the ALD (see Supplemental Material of Ref. [54]). To fully shed light on the mechanism generating the polarization dependence, experiments with different orientations of the polarizations of the pump and the probe beams need to be performed. This is a subject of future studies. However, independently of the outcome of the future suggested experiments, this discussion seems to be irrelevant in $\mathrm{K}_{2} \mathrm{NiF}_{4}$. The ISRS results for $\mathrm{K}_{2} \mathrm{NiF}_{4}$ displayed in Fig. 9 are significantly different from the $\mathrm{KNiF}_{3}$ case: the phase of the oscillations never changes regardless of the polarization of the pump beam. We note that the measurements performed with circularly and linearly polarized light are two different experiments, since different waveplates are employed and thus the optical alignment differs as well. Consequently, experiments with linearly and circularly polarized light cannot be compared.

From Eq. (60), it follows that the symmetry allows different values of the generalized force $H_{\mu}$ for the parallel $\left(e_{\|}=1, \quad e_{\perp}=0\right)$ and perpendicular $\left(e_{\|}=0\right.$, $e_{\perp}=1$ ) configurations. Considering Eq. (60), we can then formulate the following predictions. (1) if light propagates along the Néel vector, as in the case of $\mathrm{K}_{2} \mathrm{NiF}_{4}, e_{\|}=0$, $\Delta L \propto H_{\mu} \propto\left(\chi_{13}-\chi_{11}\right)$ and does not depend upon the direction of light polarization. (2) If light propagates perpendicularly to the Néel vector, as in the case of $\mathrm{KNiF}_{3}$, both $e_{\|}$and $e_{\perp}$ components of the polarization vector could be nonzero. The difference between two orthogonal polarization states $\mathbf{E} \|\left(e_{\|}, e_{\perp}\right)$ and $\mathbf{E} \|\left(-e_{\perp}, e_{\|}\right)$ is $\propto\left(\chi_{33}-\chi_{31}-\chi_{13}+\chi_{11}\right)\left(e_{\|}^{2}-e_{\perp}^{2}\right)$. It is maximal

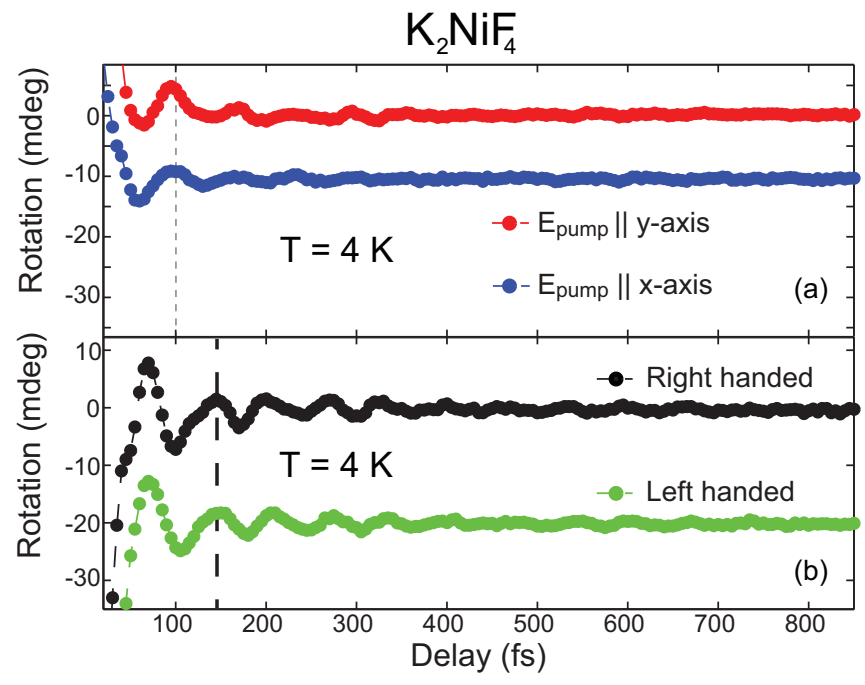

FIG. 9. The measurements of $\mathrm{K}_{2} \mathrm{NiF}_{4}$ were performed at $4 \mathrm{~K}$. The pump and probe photon energies were 1.9 and $1.3 \mathrm{eV}$, respectively. The grey and black dashed lines highlight the phase relation among the different time traces in the case of linearly or circularly polarized pump beam, respectively. The experimental geometry is shown in Fig. 4(b). (a) The excitation beam was linearly polarized and the pump fluence was $\approx 3 \mathrm{~mJ} / \mathrm{cm}^{2}$. (b) The data here shown were obtained by employing circularly polarized pump beams. The fluence was $\approx 4 \mathrm{~mJ} / \mathrm{cm}^{2}$.

when light is polarized parallel/perpendicular to the Néel vector.

In accordance with the symmetry analysis, no polarization dependence was observed in $\mathrm{KNi}_{2} \mathrm{~F}_{4}$ if the exciting laser pump beam propagates along the antiferromagnetic vector. It may be surprising that the oscillations are observed at all. According to our model the antiferromagnetic order is probed due to ALD, which in the case of $\mathrm{K}_{2} \mathrm{NiF}_{4}$ should be zero, if light propagates along the antiferromagnetic vector. The apparent contradiction is explained by the fact that during the experiment the probe and the pump beams were impinging on the sample at different angles. Therefore, if the pump was propagating nearly along the antiferromagnetic vector, the wave vector of the probe was at an angle to $\mathbf{L}$ and magnetic linear dichroism was allowed. To verify this hypothesis experiments with different orientations of the crystal are necessary. Such experiments are subject of future studies.

Moreover, analogously to the case of $\mathrm{KNiF}_{3}$, symmetry arguments determine that a purely circularly polarized beam cannot generate coherent femto-nanomagnons in $\mathrm{K}_{2} \mathrm{NiF}_{4}$ as well. However, in addition to the aforementioned observation concerning the purity of the polarization state of our laser beams, we would like to remind that static magneto-optical effects induce further distortions of the polarization. Considering the remarkable thicknesses of our samples (in particular of $\mathrm{K}_{2} \mathrm{NiF}_{4}$ ), the magneto-optical effects, which are proportional to the propagation distance of light in a magnetic material, play a non-negligible role in modifying the polarization of the laser beams. Therefore a magnonic signal was detected also by illuminating the sample with circularly polarized laser pulses [Fig. 9(b)]. 

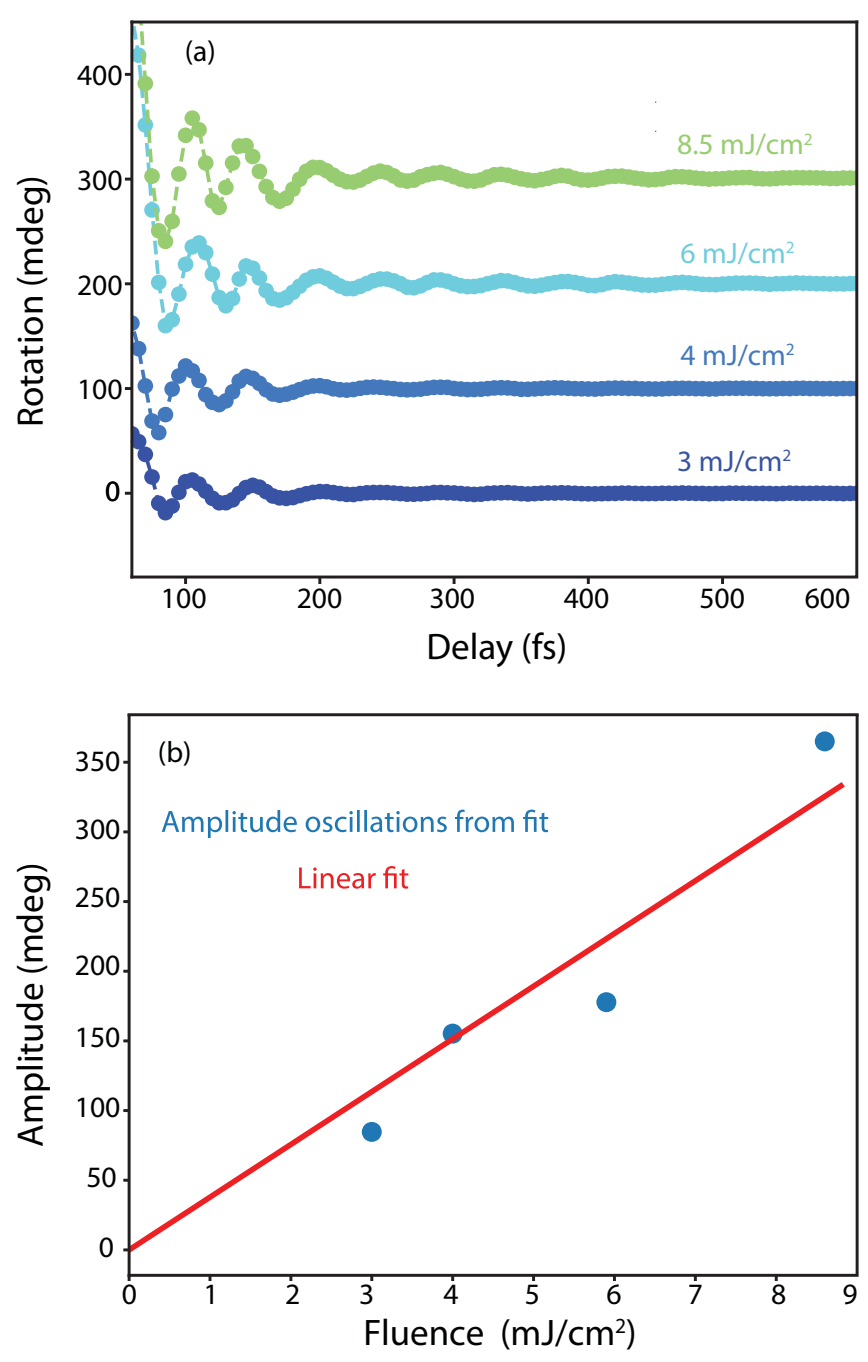

FIG. 10. The measurements were performed on $\mathrm{KNiF}_{3}$ at $77 \mathrm{~K}$. (a) Time dependence of the photoinduced spin dynamics for several pump fluences. The time traces are shifted vertically for presentation purposes. (b) The time traces were fitted with a damped harmonic function in order to retrieve the amplitude of the mode. The dependence of the amplitude of the oscillations on the excitation fluence can be described by a linear function (obtained via fitting); most importantly no trace of a quadratic trend is visible.

\section{PUMP FLUENCE DEPENDENCE}

The dependence of the spin dynamics on the excitation fluence was investigated. This experiment was performed on $\mathrm{KNiF}_{3}$ at liquid nitrogen temperature. The pump and probe photon energies were 1.9 and $1.3 \mathrm{eV}$ respectively; both beam were linearly polarized. The results, shown in Fig. 10, reveal that the amplitude of the magnonic signal is enhanced as the excitation intensity increases. Moreover, we estimated the amplitude of the magnonic signal by fitting the time traces with a damped harmonic function. The result of this procedure is shown in Fig. 10(b), in which the fluence dependence of the amplitude of the oscillations is shown. The obtained trend is consistent with a linear function. Importantly, no clear signatures of quadratic or even higher-order effects are visible. This provides evidence that a classical description of two-magnon dynamics is not adequate, since it predicts spin oscillations scaling quadratically with the pump intensity, as discussed in Sec. II and reported [28]. Consequently, even the magneto-optical detection has to rely on effects linearly proportional to the intensity of light, as reported in Eq. (67).

\section{CONCLUSIONS AND PERSPECTIVES}

The present experimental and theoretical results provide a comprehensive description of the spin dynamics triggered by the ISRS excitation of femto-nanomagnons. We have experimentally demonstrated that, despite the vanishing wave vector exchanged during the light-matter interaction, only spin excitations near the edges of the Brillouin zone are actually involved. This conclusion is confirmed even further noticing that consistent results were obtained in antiferromagnets with different magnetocrystalline anisotropy (cubic$\mathrm{KNiF}_{3}$ and uniaxial $\mathrm{K}_{2} \mathrm{NiF}_{4}$ ). The data allowed also to derive a criterion for the experimental conditions required to achieve the phase control of the magnons. The femto-nanomagnonic regime here reported is a unique case of light-induced spin dynamics which cannot be interpreted in the realm of classical physics. The coherent longitudinal oscillations of the antiferromagnetic vector triggered by laser pulses demand a quantum mechanical approach. Formulating a microscopic description in terms of perturbations of exchange interactions and magnon pair operators, we employ coherent states for these operators to provide an effectively macroscopic equation of motion beyond thermodynamics for the coherent longitudinal dynamics of the antiferromagnetic vector. Our model predicts that the pairs of magnons involved in this process are entangled, which may be verified in a nonlocal experimental scheme. This fact, combined with the achievement of the conditions required to manipulate the phase of the magnonic oscillations, may pave the way to a concept of femtosecond manipulation of the entanglement in solid state compounds aimed at ultrafast quantum information technology. We want to stress that no classical counterpart of this peculiar spin dynamical regime exists.

Although our experiments have been limited to model systems, the approach can be extended to several classes of materials. For instance, pumping the $2 \mathrm{M}$ mode in ferrimagnets and weak antiferromagnets would address the fascinating question of the role played by the magnetization in the highest-possible frequency spin dynamics. Time-resolved experiments in such materials could photoinduce and manipulate the ultimately fastest dynamics of the angular momentum. It is also worth to mention that femto-nanomagnons have been hitherto studied exclusively in collinear spin structures. Different systems, especially spin textures with topological objects like bubbles, skyrmions, and domain walls with a size comparable with the wavelength of the femto-nanomagnons, could be dramatically affected by the photoexcitation of magnons near the edges of the Brillouin zone. Another enticing perspective consists in exciting resonantly the femto-nanomagnons. Although a direct ultrafast excitation of the $2 \mathrm{M}$ mode is far from trivial and unexplored so far, a first demonstration of resonant pumping of magnons via a different mechanism has recently been reported to be able even to induce magnetic phase transitions on the femtosecond timescale [61]. 


\section{ACKNOWLEDGMENTS}

The research leading to this results was supported by the Japanese Society for Promotion of Science (JSPS) "Postdoctoral Fellowship for Overseas Researcher" No. P16326, de Nederlandse Organisatie voor Wetenschappelijk Onderzoek (NWO), de Stichting voor Fundamenteel Onderzoek der Materie (FOM), ERC Advanced Grant No. 339813 (EXCHANGE), LASERLAB-EUROPE (Grants Agreement No. 284464 and No. 654148, EC's Seventh Framework Programme). R.V.P. acknowledges the support from the Russian Science Foundation, Project No. 16-12-10456. J.H.M. acknowledges funding by the Nederlandse Organisatie voor Wetenschappelijk Onderzoek (NWO) by a VENI grant, by the European Research Council (ERC) Advanced Grant No. 338957 (FEMTO/NANO) and is part of the ShellNWO/FOM-initiative "Computational sciences for energy research" of Shell and Chemical Sciences, Earth and Life Sciences, Physical Sciences, FOM and STW. H.G. and J.S. acknowledge support from Humboldt Foundation, EU ERC Advanced Grant No. 268066 and the Transregional Collaborative Research Center (SFB/TRR) 173 SPIN+X. G.C. acknowledges support by the European Union Horizon 2020 Programme under Grant Agreement No. 696656 Graphene Flagship.

D.B., S.D.C., J.H.M., and O.G. equally contributed to this work.

\section{APPENDIX A}

In this Appendix, we show how $H_{0}$ and $\delta H$ [Eqs. (3) and (5) of the main text] can be written in terms of magnon annihilation (creation) operators $\hat{\alpha}_{\mathbf{k}}\left(\hat{\alpha}_{\mathbf{k}}^{\dagger}\right)$ and $\hat{\beta}_{\mathbf{k}}\left(\hat{\beta}_{\mathbf{k}}^{\dagger}\right)$. Following the literature $[35,62]$, our approach consists in computing weak deviations from the classical Néel state, which we express by introducing Holstein-Primakoff bosons for sublattice $A$ and $B$ :

$$
\begin{aligned}
& S_{A i}^{+}=\sqrt{2 S}\left(1-\frac{a_{i}^{\dagger} a_{i}}{2 S}\right)^{1 / 2} a_{i}, \quad S_{A i}^{-}=\sqrt{2 S} a_{i}^{\dagger}\left(1-\frac{a_{i}^{\dagger} a_{i}}{2 S}\right)^{1 / 2}, \\
& S_{A i}^{z}=S-a_{i}^{\dagger} a_{i}, \\
& S_{B i}^{+}=\sqrt{2 S} b_{i}^{\dagger}\left(1-\frac{b_{i}^{\dagger} b_{i}}{2 S}\right)^{1 / 2}, \quad S_{B i}^{-}=\sqrt{2 S}\left(1-\frac{b_{i}^{\dagger} b_{i}}{2 S}\right)^{1 / 2} b_{i}, \\
& S_{B i}^{z}=-S+b_{i}^{\dagger} b_{i} .
\end{aligned}
$$

Attempting to obtain magnon states, we introduce the Fourier transforms:

$$
\begin{array}{ll}
a_{\mathbf{k}}^{\dagger}=\sqrt{\frac{2}{N}} \sum_{i} e^{-\mathrm{i} \mathbf{k} \cdot \mathbf{R}_{i}} a_{i}^{\dagger} & a_{i}^{\dagger}=\sqrt{\frac{2}{N}} \sum_{\mathbf{k}} e^{\mathrm{ik} \cdot \mathbf{R}_{i}} a_{\mathbf{k}}^{\dagger}, \\
b_{\mathbf{k}}^{\dagger}=\sqrt{\frac{2}{N}} \sum_{i} e^{-\mathrm{i} \mathbf{k} \cdot \mathbf{R}_{i}} b_{i}^{\dagger} & b_{i}^{\dagger}=\sqrt{\frac{2}{N}} \sum_{\mathbf{k}} e^{\mathrm{ik} \cdot \mathbf{R}_{i}} b_{\mathbf{k}}^{\dagger} .
\end{array}
$$

Here the factor $\sqrt{2 / N}$ appears since the magnetic Brillouin zone contains only $N / 2 \mathbf{k}$ vectors, with $N$ the number of sites in the original lattice. After these transformations, the harmonic part of $H_{0}$ becomes

$$
\begin{aligned}
H_{0} \approx & -\frac{z_{N} J N}{2} S^{2}+z_{N} J S \sum_{\mathbf{k}}\left[\gamma_{\mathbf{k}}\left(a_{\mathbf{k}} b_{-\mathbf{k}}+b_{\mathbf{k}}^{\dagger} a_{-\mathbf{k}}^{\dagger}\right)\right. \\
& \left.+\left(a_{\mathbf{k}}^{\dagger} a_{\mathbf{k}}+b_{\mathbf{k}}^{\dagger} b_{\mathbf{k}}\right)\right],
\end{aligned}
$$

where $\gamma_{\mathbf{k}}=\frac{1}{z_{N}} \sum_{\delta} \exp (i \mathbf{k} \cdot \boldsymbol{\delta})$ depends on the geometry of the exchange bonds given the sum over nearest-neighbor bonds $\delta$. Due to the nearest-neighbor coupling between the sublattices $A, B$, the operators $a_{\mathbf{k}}, b_{\mathbf{k}}$ do not diagonalize $H_{0}$. The physical magnons therefore comprise superpositions of $a_{\mathbf{k}}$ and $b_{-\mathbf{k}}^{\dagger}$ as described by the Bogoliubov transformation:

$$
\begin{aligned}
& a_{\mathbf{k}}=u_{\mathbf{k}} \alpha_{\mathbf{k}}+v_{\mathbf{k}} \beta_{-\mathbf{k}}^{\dagger}, \\
& b_{\mathbf{k}}=u_{\mathbf{k}} \beta_{\mathbf{k}}+v_{\mathbf{k}} \alpha_{-\mathbf{k}}^{\dagger} .
\end{aligned}
$$

Requiring the Bose commutation relations $\left[\alpha_{\mathbf{k}}, \alpha_{\mathbf{k}}^{\dagger}\right]=1$, $\left[\beta_{\mathbf{k}}, \beta_{\mathbf{k}}^{\dagger}\right]=1$ implies $u_{\mathbf{k}}^{2}-v_{\mathbf{k}}^{2}=1$ and we can choose symmetric and real coefficients $u_{\mathbf{k}}=u_{-\mathbf{k}}, v_{\mathbf{k}}=v_{-\mathbf{k}}$, yielding

$$
\begin{aligned}
H_{0} \approx & -\frac{z_{N} J N}{2} S(S+1) \\
& +z_{N} J S \sum_{\mathbf{k}}\left(u_{\mathbf{k}}^{2}+u_{\mathbf{k}}^{2}+2 u_{\mathbf{k}} v_{\mathbf{k}} \gamma_{\mathbf{k}}\right)\left[\alpha_{\mathbf{k}}^{\dagger} \alpha_{\mathbf{k}}+\beta_{\mathbf{k}}^{\dagger} \beta_{\mathbf{k}}+1\right] \\
& +z_{N} J S \sum_{\mathbf{k}}\left(\gamma_{\mathbf{k}}\left(u_{\mathbf{k}}^{2}+v_{\mathbf{k}}^{2}\right)+2 u_{\mathbf{k}} v_{\mathbf{k}}\right)\left[\alpha_{\mathbf{k}} \beta_{-\mathbf{k}}+\alpha_{\mathbf{k}}^{\dagger} \beta_{-\mathbf{k}}^{\dagger}\right]
\end{aligned}
$$

The coefficients $u_{\mathbf{k}}, v_{\mathbf{k}}$ of the transformation are now chosen such that the off-diagonal terms vanish:

$$
\gamma_{\mathbf{k}}\left(u_{\mathbf{k}}^{2}+v_{\mathbf{k}}^{2}\right)+2 u_{\mathbf{k}} v_{\mathbf{k}}=0
$$

yielding the diagonal form

$$
\begin{aligned}
& H_{0} \approx-\frac{z_{N} J N}{2} S(S+1)+\sum_{\mathbf{k}} \hbar \omega_{\mathbf{k}}\left[\alpha_{\mathbf{k}}^{\dagger} \alpha_{\mathbf{k}}+\beta_{\mathbf{k}}^{\dagger} \beta_{\mathbf{k}}+1\right] \\
& \hbar \omega_{\mathbf{k}} \equiv z_{N} J S\left(u_{\mathbf{k}}^{2}+v_{\mathbf{k}}^{2}+2 u_{\mathbf{k}} v_{\mathbf{k}} \gamma_{\mathbf{k}}\right)=z_{N} J S \sqrt{1-\gamma_{\mathbf{k}}^{2}} .
\end{aligned}
$$

Next, we similarly transform the light-induced perturbation $\delta H$. This produces equivalent results with the replacements

$$
J \rightarrow \Delta J, \quad \gamma_{\mathbf{k}} \rightarrow \xi_{\mathbf{k}}=\frac{1}{z} \sum_{\delta}(\hat{e} \cdot \hat{\boldsymbol{\delta}})^{2} \exp (i \mathbf{k} \cdot \boldsymbol{\delta}) .
$$

Using Eq. (A8), we write this as

$$
\begin{aligned}
\delta H \approx & -\frac{z_{N} \Delta J N}{2} S(S+1)+\sum_{\mathbf{k}} \hbar \delta \omega_{\mathbf{k}}\left[\alpha_{\mathbf{k}}^{\dagger} \alpha_{\mathbf{k}}+\beta_{\mathbf{k}}^{\dagger} \beta_{\mathbf{k}}+1\right] \\
& +\hbar V_{\mathbf{k}}\left[\alpha_{\mathbf{k}} \beta_{-\mathbf{k}}+\alpha_{\mathbf{k}}^{\dagger} \beta_{-\mathbf{k}}^{\dagger}\right],
\end{aligned}
$$

where

$$
\hbar \delta \omega_{\mathbf{k}}=z_{N} \Delta J S \frac{\left(1-\gamma_{\mathbf{k}} \xi_{\mathbf{k}}\right)}{\sqrt{1-\gamma_{\mathbf{k}}^{2}}}, \quad \hbar V_{\mathbf{k}}=z_{N} \Delta J S \frac{\left(\xi_{\mathbf{k}}-\gamma_{\mathbf{k}}\right)}{\sqrt{1-\gamma_{\mathbf{k}}^{2}}} .
$$

Hence we observe that, in general, $H_{0}$ and $\delta H_{0}$ cannot be simultaneously diagonalized. Therefore, under the presence light-induced perturbations to the exchange interactions, the 
terms $\alpha_{\mathbf{k}} \beta_{-\mathbf{k}}+\alpha_{\mathbf{k}}^{\dagger} \beta_{-\mathbf{k}}^{\dagger}$ remain which can annihilate or create magnon pairs with opposite $\mathbf{k}$.

We note that there is a subtle technical difference between the magnons described by the Bose operators $a_{\mathbf{k}}, b_{\mathbf{k}}$ and those described by $\alpha_{\mathbf{k}}, \beta_{\mathbf{k}}$. The former represent excitation with respect to the collinear state (classical Néel state) and are strictly localized to one magnetic sublattice, while the latter describe excitations that are a superposition of magnons in different sublattices with respect to the ground state, which is already dressed by two-magnon excitations. Mathematically, it is convenient to work with $\alpha_{\mathbf{k}}, \beta_{\mathbf{k}}$, since they diagonalize $H_{0}$. However, it is equally possible to keep working in the basis $a_{\mathbf{k}}, b_{\mathbf{k}}$ of magnons that are completely localized to either of the magnetic sublattices. Here we elaborate further on the difference between the two representations. First of all, we stress that $\alpha_{\mathbf{k}}, \beta_{\mathbf{k}}$ almost coincide with $a_{\mathbf{k}}$ and $b_{\mathbf{k}}$ when $\mathbf{k}$ is close to the BZ boundary where $v_{\mathbf{k}} \ll u_{\mathbf{k}}$ in Eqs. (A5) and (A6). Hence, for many qualitative discussions, it is appropriate to think of $2 \mathrm{M}$ excitations as if they comprise a pair of magnons, one in each sublattice, each with the same frequency but with opposite $\mathbf{k}$ in the different sublattices. One such two-magnon state is depicted in Fig. 2(b), while Fig. 2(a) shows the Néel state without any magnon excitation. It is, however, important to stress that the Néel state is not the exact eigenstate of the quantum antiferromagnet (see also the discussion in Sec. II). Therefore, even in the ground state, there are quantum fluctuations due to incoherent two-magnon excitations. Hence, already at zero temperature both the Néel state $|0\rangle|0\rangle$ and the state $a_{\mathbf{k}}^{\dagger} b_{-\mathbf{k}}^{\dagger}|0\rangle|0\rangle$ with two magnons excited have nonzero occupation, despite the fact that the energy separation between these states is $\Delta E \sim 2 J$. A sudden perturbation $\Delta J$, therefore can induce coherent dynamics between the Néel state and the two-magnon excited state.

Using the transformed operators $\alpha_{\mathbf{k}}, \beta_{\mathbf{k}}$ a conceptually different picture arises. Since the operators $\alpha_{\mathbf{k}}, \beta_{\mathbf{k}}$ diagonalize the Hamiltonian, the excited state $\alpha_{\mathbf{k}}^{\dagger} \beta_{-\mathbf{k}}^{\dagger}|0\rangle|0\rangle$ is not occupied in the ground state. In this basis, the sudden perturbation of $J$ induces a coherence between the ground state $|0\rangle|0\rangle$ and excited state $\alpha_{\mathbf{k}}^{\dagger} \beta_{-\mathbf{k}}^{\dagger}|0\rangle|0\rangle$ that remains after the pulse, see the discussion in Ref. [63]. Of course, since the transformation between the two representations is unitary, both yield the same observables. For the qualitative discussion in
Sec. II we mostly rely on the description using the $a_{\mathbf{k}}, b_{\mathbf{k}}$ operators, since in this basis magnons are easier to visualize. On the other hand, for the theoretical derivations in Sec. II, we use the magnons $\alpha_{\mathbf{k}}, \beta_{\mathbf{k}}$ since they are more convenient mathematically.

\section{APPENDIX B: SPIN CORRELATIONS AND NÉEL VECTOR}

Here we derive the expressions for spin correlations and Néel vector within harmonic magnon theory, using the same approach as introduced above. For the components of the Néel vector, we obtain

$$
\begin{gathered}
L^{z}=N S-\sum_{i \in \Uparrow} a_{i}^{\dagger} a_{i}+\sum_{j \in \Downarrow} b_{j}^{\dagger} b_{j} \\
=N S-\sum_{\mathbf{k}} a_{\mathbf{k}}^{\dagger} a_{\mathbf{k}}+b_{\mathbf{k}}^{\dagger} b_{\mathbf{k}}, \\
L^{x}=\sqrt{N S}\left(a_{0}+a_{0}^{\dagger}-\left(b_{0}+b_{0}^{\dagger}\right)\right), \\
L^{y}=-i \sqrt{N S}\left(a_{0}-a_{0}^{\dagger}-\left(b_{0}-b_{0}^{\dagger}\right)\right) .
\end{gathered}
$$

Longitudinal spin correlations are defined as

$$
\begin{aligned}
\sum_{\langle i, j\rangle} S_{i}^{z} S_{j}^{z} & =-N z S^{2} / 2+S \sum_{i, \delta} a_{i}^{\dagger} a_{i}+b_{i+\delta}^{\dagger} b_{i+\delta} \\
& =-N z_{N} S^{2} / 2+z_{N} S \sum_{\mathbf{k}} a_{\mathbf{k}}^{\dagger} a_{\mathbf{k}}+b_{\mathbf{k}}^{\dagger} b_{\mathbf{k}} .
\end{aligned}
$$

From this we observe the direct connection between $\left\langle L^{z}\right\rangle$ and $\sum_{\langle i, j\rangle}\left\langle S_{i}^{z} S_{j}^{z}\right\rangle$ (see also Ref. [23]):

$$
L^{z}=\frac{N S}{2}-\frac{1}{z_{N} S} \sum_{\langle i, j\rangle} S_{i}^{z} S_{j}^{z} .
$$

Substitution of the Bogoliubov transformation gives for the longitudinal correlations

$$
\begin{aligned}
\sum_{\langle i, j\rangle} S_{i}^{z} S_{j}^{z}= & -N z S^{2} / 2+z S \sum_{\mathbf{k}}\left(u_{\mathbf{k}}^{2}+v_{\mathbf{k}}^{2}\right)\left(\alpha_{\mathbf{k}}^{\dagger} \alpha_{\mathbf{k}}+\beta_{-\mathbf{k}}^{\dagger} \beta_{-\mathbf{k}}+1\right) \\
& +2 u_{\mathbf{k}} v_{\mathbf{k}}\left(\alpha_{\mathbf{k}} \beta_{-\mathbf{k}}+\alpha_{\mathbf{k}}^{\dagger} \beta_{-\mathbf{k}}^{\dagger}\right) .
\end{aligned}
$$

Direct substitution of the magnon pair operators and using Eqs. (A8) and (A10) gives the formula for the longitudinal correlations (24) of the main text.
[1] E. Beaurepaire, J. C. Merle, A. Daunois, and J. Y. Bigot, Phys. Rev. Lett. 76, 4250 (1996).

[2] M. van Kampen, C. Jozsa, J. T. Kohlhepp, P. LeClair, L. Lagae, W. J. M. de Jonge, and B. Koopmans, Phys. Rev. Lett. 88, 227201 (2002).

[3] A. V. Kimel, A. Kirilyuk, A. Tsvetkov, R. V. Pisarev, and T. Rasing, Nature (London) 429, 850 (2004).

[4] A. V. Kimel, A. Kirilyuk, P. A. Usachev, R. V. Pisarev, A. M. Balbashov, and T. Rasing, Nature (London) 435, 655 (2005).

[5] T. Satoh, S.-J. Cho, R. Iida, T. Shimura, K. Kuroda, H. Ueda, Y. Ueda, B. A. Ivanov, F. Nori, and M. Fiebig, Phys. Rev. Lett. 105, 077402 (2010).
[6] N. Tesarova, P. Nemec, E. Rozkotova, J. Zemen, T. Janda, D. Butkovicova, F. Trojanek, K. Olejnik, V. Novak, P. Maly, and T. Jungwirth, Nat. Photonics 7, 492 (2013).

[7] C. D. Stanciu, F. Hansteen, A. V. Kimel, A. Kirilyuk, A. Tsukamoto, A. Itoh, and T. Rasing, Phys. Rev. Lett. 99, 047601 (2007).

[8] T. Satoh, Y. Terui, R. Moriya, B. A. Ivanov, K. Ando, E. Saitoh, T. Shimura, and K. Kuroda, Nat. Photonics 6, 662 (2012).

[9] Y. Hashimoto, S. Daimon, Y. Oikawa, K. Shen, K. Sato, D Bossini, Y. Tabuchi, T. Satoh, B. Hillebrands, G. E. W. Bauer, T. H. Johansen, A. Kirilyuk, Th. Rasing, and E. Saitoh, Nat. Commun. 8, 15859 (2017). 
[10] Y. Hashimoto, D. Bossini, T. H. Johansen, E. Saitoh, A. Kirilyuk, and Th. Rasing, Phys. Rev. B 97, 140404(R) (2018).

[11] B. Lenk, H. Ulrichs, and M. Münzenberg, Phys. Rep. 507, 107 (2011).

[12] T. Li, A. Patz, L. Mouchlidas, J. Yan, T. Lograsso, I. E. Perakis, and J. Wang, Nature (London) 496, 69 (2013).

[13] I. Radu, K. Vahaplar, C. Stamm, T. Kachel, N. Pontius, H. A. Dürr, T. A. Ostler, J. Barker, R. F. L. Evans, R. W. Chantrell, A. Tsukamoto, A. Itoh, A. Kirilyuk, T. Rasing, and A. V. Kimel, Nature (London) 472, 205 (2011).

[14] D. Bossini, A. M. Kalashnikova, R. V. Pisarev, T. Rasing, and A. V. Kimel, Phys. Rev. B 89, 060405(R) (2014).

[15] A. M. Kalashnikova, A. V. Kimel, R. V. Pisarev, V. N. Gridnev, P. A. Usachev, A. Kirilyuk, and T. Rasing, Phys. Rev. B 78, 104301 (2008).

[16] A. M. Kalashnikova, A. V. Kimel, R. V. Pisarev, V. N. Gridnev, A. Kirilyuk, and T. Rasing, Phys. Rev. Lett. 99, 167205 (2007).

[17] R. Loudon and P. A. Fleury, Phys. Rev. 166, 514 (1968).

[18] S. Chinn, H. J. Zeiger, and J. R. O’Connor, Phys. Rev. B 3, 1709 (1971).

[19] P. A. Fleury and H. J. Guggenheim, Phys. Rev. Lett. 24, 1346 (1970).

[20] U. Balucani and V. Tognetti, Phys. Rev. B 8, 4247 (1973).

[21] M. G. Cottam and D. J. Lockwood, Light Scattering in Magnetic Solids, 1st ed. (Wiley-Interscience, New York, 1986).

[22] P. Lemmens, G. Güntherodt, and C. Gros, Phys. Rep. 375, 1 (2003).

[23] D. Bossini, S. Dal Conte, Y. Hashimoto, A. Secchi, R. V. Pisarev, T. Rasing, G. Cerullo, and A. V. Kimel, Nat. Commun. 7, 10645 (2016).

[24] J. Zhao, A. V. Bragas, D. J. Lockwood, and R. Merlin, Phys. Rev. Lett. 93, 107203 (2004).

[25] J. Zhao, A. V. Bragas, R. Merlin, and D. J. Lockwood, Phys. Rev. B 73, 184434 (2006).

[26] A. Kirilyuk, A. V. Kimel, and T. Rasing, Rev. Mod. Phys. 82, 2731 (2010).

[27] L. D. Landau and E. M. Lifshitz, Electrodynamics of Continuous Media, 2nd ed. (Pergamon, New York, 1984).

[28] S. Baierl, J. H. Mentink, M. Hohenleutner, L. Braun, T.-M. Do, C. Lange, A. Sell, M. Fiebig, G. Woltersdorf, T. Kampfrath, and R. Huber, Phys. Rev. Lett. 117, 197201 (2016).

[29] J. Hellsvik, J. H. Mentink, and J. Lorenzana, Phys. Rev. B 94, 144435 (2016).

[30] M. E. Lines, Phys. Rev. 164, 736 (1967).

[31] D. J. Lockwood and M. G. Cottam, Phys. Rev. B 35, 1973 (1987).

[32] J. Mentink, K. Balzer, and M. Eckstein, Nat. Commun. 6, 6708 (2015).

[33] R. V. Mikhaylovskiy, E. Hendry, A. Secchi, J. H. Mentink, M. Eckstein, A. Wu, R. V. Pisarev, V. V. Kruglyak, M. I. Katsnelson, T. Rasing, and A. V. Kimel, Nat. Commun. 6, 8190 (2015).

[34] A. P. Itin and M. I. Katsnelson, Phys. Rev. Lett. 115, 075301 (2015).

[35] P. Fazekas, Lecture Notes on Electron Correlation and Magnetism (World Scientific, Singapore, 1999).
[36] A. M. Perelomov, Commun. Math. Phys. 44, 197 (1975).

[37] D. C. Mattis, Electron Correlation and Magnetism in NarrowBand Systems, Springer Series in Solid-State Sciences Vol. 17 (Springer, Berlin, Heidelberg, 1981), pp. 126.

[38] M. Novaes, Rev. Bras. Ensino Fis. 26, 351 (2004).

[39] A. Bechler, J. Phys. A: Math. Gen. 34, 8081 (2001).

[40] D. Money, D. Paige, W. Corner, and B. Tanner, J. Magn. Magn. Mater. 15-18, 603 (1980).

[41] F. Ganot, C. Dugautier, P. Moch, and J. Nouet, J. Phys. C Solid State Phys. 15, 801 (1982).

[42] R. R. Birss, Symmetry and Magnetism (Wiley, New York, 1966).

[43] V. C. Usenko and M. G. A. Paris, Phys. Rev. A 75, 043812 (2007).

[44] H.-C. Fu and R. Sasaki, Phys. Rev. A 53, 3836 (1996).

[45] V. Bužek, J. Mod. Opt. 37, 303 (1990).

[46] M. Trigo, M. Fuchs, J. Chen, M. P. Jiang, M. Cammarata, S. Fahy, D. M. Fritz, K. Gaffney, S. Ghimire, A. Higginbotham, S. L. Johnson, M. E. Kozina, J. Larsson, H. Lemke, A. M. Lindenberg, G. Ndabashimiye, F. Quirin, K. SokolowskiTinten, C. Uher, G. Wang, J. S. Wark, D. Zhu, and D. A. Reis, Nat. Phys. 9, 790 (2013).

[47] S. L. Johnson, P. Beaud, E. Vorobeva, C. J. Milne, É. D. Murray, S. Fahy, and G. Ingold, Phys. Rev. Lett. 102, 175503 (2009).

[48] D. Bossini, V. I. Belotelov, A. K. Zvezdin, A. N. Kalish, and A. V. Kimel, ACS Photonics 3, 1385 (2016).

[49] D. Bossini and T. Rasing, Phys. Scr. 92, 024002 (2017).

[50] D. Brida, C. Manzoni, G. Cirmi, M. Marangoni, S. Bonora, P. Villoresi, S. De Silvestri, and G. Cerullo, J. Opt. 12, 013001 (2009).

[51] S. Dal Conte, D. Brida, A. Damascelli, F. Banfi, H. Eisaki, L. Vidmar, D. Golež, M. Mierzejewski, G. Soavi, S. Peli, G. Ferrini, R. Comin, B. M. Ludbrook, L. Chauviere, N. D. Zhigadlo, M. Greven, S. Lupi, M. Capone, J. Bonča, G. Cerullo, and C. Giannetti, Nat. Phys. 11, 421 (2015).

[52] G. A. Smolenskii, R. V. Pisarev, and G. Sinii, Sov. Phys.-USP 18, 410 (1976).

[53] J. Ferré and G. A. Gehring, Rep. Prog. Phys. 47, 513 (1984).

[54] V. Saidl, P. Nemec, P. Wadley, V. Hills, R. P. Campion, V. Novak, K. W. Edmonds, F. Maccherozzi, S. S. Dhesi, B. L. Gallagher, F. Trojanek, J. Kunes, J. Zelezny, P. Maly, and T. Jungwirth, Nat. Photon 11, 91 (2017).

[55] A. T. Abdalian and P. Moch, J. Phys. C 21, 767 (2000).

[56] M. Safa and B. K. Tanner, Philos. Mag. B 37, 739 (1978).

[57] D. J. Toms, W. J. O’Sullivan, and H. J. Guggenheim, Solid State Commun. 14, 715 (1974).

[58] N. D. Mermin and H. Wagner, Phys. Rev. Lett. 17, 1133 (1966).

[59] R. J. Birgeneau, F. DeRosa, and H. J. Guggenheim, Solid State Commun. 8, 13 (1970).

[60] A. K. Zvezdin and V. A. Kotov, Modern Magneetooptics and Magnetooptical Materials (IOP, Bristol, 1997).

[61] D. Bossini, K. Konishi, S. Toyoda, T. Arima, J. Yumoto, and M. Kuwata-Gonokami, Nat. Phys. 14, 370 (2018).

[62] C. Kittel, Quantum Theory of Solids, 1st ed. (Wiley, New York, N.Y., 1963).

[63] J. H. Mentink, J. Phys.: Condens. Matter 29, 453001 (2017). 